\title{
THE INFRARED SPECTRAL PROPERTIES OF MAGELLANIC CARBON STARS
}

\author{
G. C. Sloan ${ }^{1,2}$, K. E. Kraemer ${ }^{3}$, I. McDonald ${ }^{4}$, M. A. T. Groenewegen ${ }^{5}$, P. R. Wood ${ }^{6}$, A. A. Zijlstra ${ }^{4}$,

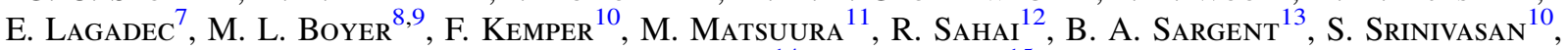 \\ J. TH. VAN LOON ${ }^{14}$, AND K. VOLK ${ }^{15}$ \\ ${ }^{1}$ Cornell Center for Astrophysics \& Planetary Science, Cornell Univ., Ithaca, NY 14853-6801, USA; sloan @isc.astro.cornell.edu \\ ${ }^{2}$ Department of Physics and Astronomy, University of North Carolina, Chapel Hill, NC 27599-3255, USA \\ ${ }^{3}$ Institute for Scientific Research, Boston College, 140 Commonwealth Avenue, Chestnut Hill, MA 02467, USA \\ ${ }^{4}$ Jodrell Bank Centre for Astrophysics, Univ. of Manchester, Manchester M13 9PL, UK \\ ${ }^{5}$ Koninklijke Sterrenwacht van België, Ringlaan 3, B-1180 Brussels, Belgium \\ ${ }^{6}$ Research School of Astronomy and Astrophysics, Australian National University, Canberra, ACT 2611, Australia \\ ${ }^{7}$ Observatoire de la Côte d'Azur, F-06300, Nice, France \\ ${ }^{8}$ CRESST and Observational Cosmology Lab, Code 665, NASA Goddard Space Flight Center, Greenbelt, MD, 20771, USA \\ ${ }^{9}$ Department of Astronomy, University of Maryland, College Park, MD 20742, USA \\ ${ }^{10}$ Academia Sinica, Institute of Astronomy and Astrophysics, 11F Astronomy-Mathematics Building, NTU/AS, \\ No. 1, Sec. 4, Roosevelt Rd., Taipei 10617, Taiwan, R.O.C. \\ ${ }^{11}$ School of Physics and Astronomy, Cardiff University, Queen's Buildings, The Parade, Cardiff, CF24 3AA, UK \\ ${ }^{12}$ Jet Propulsion Laboratory, California Institute of Technology, MS 183-900, Pasadena, CA 91109, USA \\ ${ }^{13}$ Center for Imaging Science and Laboratory for Multiwavelength Astrophysics, Rochester Institute of Technology, \\ 54 Lomb Memorial Drive, Rochester, NY 14623, USA \\ ${ }^{14}$ Lennard Jones Laboratories, Keele University, Staffordshire ST5 5BG, UK \\ ${ }^{15}$ Space Telescope Science Institute, 3700 San Martin Dr., Baltimore, MD 21218, USA \\ Received 2015 December 23; revised 2016 April 8; accepted 2016 April 15; published 2016 July 18
}

\begin{abstract}
The Infrared Spectrograph on the Spitzer Space Telescope observed 184 carbon stars in the Magellanic Clouds. This sample reveals that the dust-production rate (DPR) from carbon stars generally increases with the pulsation period of the star. The composition of the dust grains follows two condensation sequences, with more SiC condensing before amorphous carbon in metal-rich stars, and the order reversed in metal-poor stars. MgS dust condenses in optically thicker dust shells, and its condensation is delayed in more metal-poor stars. Metal-poor carbon stars also tend to have stronger absorption from $\mathrm{C}_{2} \mathrm{H}_{2}$ at $7.5 \mu \mathrm{m}$. The relation between DPR and pulsation period shows significant apparent scatter, which results from the initial mass of the star, with more massive stars occupying a sequence parallel to lower-mass stars, but shifted to longer periods. Accounting for differences in the mass distribution between the carbon stars observed in the Small and Large Magellanic Clouds reveals a hint of a subtle decrease in the DPR at lower metallicities, but it is not statistically significant. The most deeply embedded carbon stars have lower variability amplitudes and show $\mathrm{SiC}$ in absorption. In some cases they have bluer colors at shorter wavelengths, suggesting that the central star is becoming visible. These deeply embedded stars may be evolving off of the asymptotic giant branch and/or they may have non-spherical dust geometries.
\end{abstract}

Key words: circumstellar matter - infrared: stars - stars: AGB and post-AGB - stars: carbon

Supporting material: machine-readable tables

\section{INTRODUCTION}

Intermediate-mass stars contribute to the chemical evolution of galaxies when they shed their envelopes and seed their environments with freshly condensed dust and the products of nuclear fusion from their cores. The sensitivity of the Infrared Spectrograph (IRS; Houck et al. 2004) on the Spitzer Space Telescope (Werner et al. 2004) made it possible to observe many mass-losing stars in nearby galaxies like the Large and Small Magellanic Clouds (LMC and SMC). These stars are at known distances, and they probe different metallicities. While the metallicity distribution functions are broad enough to overlap and depend on age, for statistical treatment of large samples it is safe to claim that the LMC is more metal-poor than the Galaxy, and the SMC is more metal-poor still. As an example, Piatti \& Giesler (2013) find that in the LMC, [Fe/H] increased from $\sim-0.7$ to $\sim-0.3$ from 5 to $1 \mathrm{Gyr}$ ago, compared to $\sim-1.2$ to $\sim-0.6$ over the same time interval in the SMC (Piatti 2012).

Carbon stars dominated the spectral samples of evolved stars observed early in the Spitzer mission. Sloan et al. (2006) examined carbon stars in the SMC while Zijlstra et al. (2006) considered the LMC. Matsuura et al. (2006) studied the molecular absorption in these samples, and other studies followed (Buchanan et al. 2006; Lagadec et al. 2007; Leisenring et al. 2008; Sloan et al. 2008). Further observing programs added considerably to the spectral sample of carbon stars in both galaxies, including two programs which added fainter carbon stars to the samples in the LMC (Kemper et al. 2010) and SMC (Program 50240).

These earlier efforts led to the finding that the amount of dust observed around carbon stars does not show a strong dependence on metallicity (Sloan et al. 2008). Carbon stars result from the dredge-up of freshly fused carbon from the cores of stars as they burn $\mathrm{He}$ in thermal pulses while on the asymptotic giant branch (AGB). The formation of CO leaves only carbon or oxygen available to form other molecules and condense into dust so that when the $\mathrm{C} / \mathrm{O}$ ratio exceeds unity, the gas and dust shift from an O-rich to a C-rich chemistry. Matsuura et al. (2005) noted that because dredged-up material on the AGB is dominated by carbon in most stars, the amount 
Table 1

The Spitzer Sample of Magellanic Carbon Stars

\begin{tabular}{|c|c|c|c|c|c|c|c|}
\hline Target & Alias & R.A. & $(\mathrm{J} 2000)$ & $\begin{array}{c}\text { Position } \\
\text { Reference }\end{array}$ & $\begin{array}{l}\text { Period } \\
\text { (days) }\end{array}$ & $\begin{array}{c}\text { Period } \\
\text { Reference }^{\mathrm{a}}\end{array}$ & $\begin{array}{c}\text { Program } \\
\text { Identifier }^{\mathrm{b}}\end{array}$ \\
\hline GM 780 & $\ldots$ & 8.905255 & -73.165604 & 2MASS & 611 & OGLE & 3505 \\
\hline MSX SMC 091 & $\ldots$ & 9.236293 & -72.421547 & 2MASS & $\ldots$ & $\ldots$ & 3277 \\
\hline MSX SMC 062 & $\ldots$ & 10.670455 & -72.951599 & 2MASS & 550 & OGLE & 3277 \\
\hline MSX SMC 054 & $\ldots$ & 10.774604 & -73.361282 & 2MASS & 396 & OGLE & 3277 \\
\hline 2MASS J004326 & $\ldots$ & 10.860405 & -73.445366 & 2MASS & 330 & R05 & 50240 \\
\hline MSX SMC 044 & $\cdots$ & 10.914901 & -73.249336 & 2MASS & 440 & OGLE & 3277 \\
\hline MSX SMC 105 & $\ldots$ & 11.258941 & -72.873428 & 2MASS & 668 & OGLE & 3277 \\
\hline MSX SMC 036 & $\ldots$ & 11.474785 & -73.394775 & 2MASS & 553 & OGLE & 3277 \\
\hline GB S06 & MSX SMC 060 & 11.668442 & -73.279793 & 2MASS & 435 & OGLE & 3277 \\
\hline MSX SMC 200 & $\ldots$ & 11.711623 & -71.794250 & 2MASS & 426 & OGLE & 3277 \\
\hline
\end{tabular}

Notes.

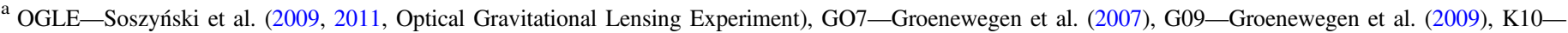

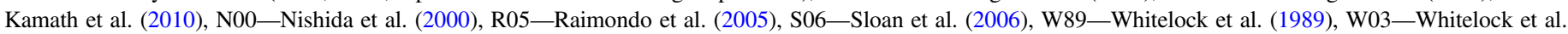
(2003), Z06-Zijlstra et al. (2006); Appendices Band C-the appendices in this paper.

${ }^{\mathrm{b}}$ See Table 2 .

(This table is available in its entirety in machine-readable form.)

of free carbon left after the formation of $\mathrm{CO}$ should actually increase in more metal-poor stars. Sloan et al. (2012) accounted for the changing oxygen abundances at lower metallicity and reached a similar conclusion. Thus, the question is not one of why the amount of carbon-rich dust is not declining as metallicity drops, but why is it not increasing.

The infrared spectrographic studies of Magellanic carbon stars have revealed several dependencies on metallicity. In more metal-poor samples, the $\mathrm{SiC}$ dust emission feature at $\sim 11.3 \mu \mathrm{m}$ weakens, the molecular absorption bands from $\mathrm{C}_{2} \mathrm{H}_{2}$ at 7.5 and $13.7 \mu \mathrm{m}$ grow stronger, and the appearance of $\mathrm{MgS}$ dust is more delayed.

This work builds on the earlier works by adding the rest of the IRS observations and comparing the samples as a whole. Section 2 describes the samples of carbon stars in the Magellanic Clouds and the Galaxy and the spectroscopic and photometric data. Section 3 describes the data analysis, and Section 4 presents the results. In Section 5, we discuss the issues raised, and in Section 6, summarize our findings. Appendix A presents useful relations between infrared filter sets and colors for carbon stars. Appendices B and C present new periods for several sources based on the analysis of multiepoch photometry in the near-IR and mid-IR, respectively.

\section{OBSERVATIONS}

\subsection{Samples and Targets}

Table 1 lists the 144 objects in the LMC and 40 in the SMC observed with the IRS and identified as carbon stars. Our objective was to build a sample including all of the carbon-rich AGB stars observed by the IRS while excluding post-AGB objects. Our selection criteria are based on the infrared (IR) spectral classification of Kraemer et al. (2002, see Section 2.5 below). Generally, spectra are considered carbon-rich if they show $\mathrm{SiC}$ dust emission at $\sim 11.3 \mu \mathrm{m}$ or the acetylene absorption bands at 7.5 and/or $13.7 \mu \mathrm{m}$. The $26-30 \mu \mathrm{m}$ feature attributed to $\mathrm{MgS}$ dust appears in many of the spectra and helps to confirm their carbon-rich nature.

Goebel \& Moseley (1985) identified MgS dust as the carrier of the 26-30 $\mu \mathrm{m}$ feature, but some doubt has been cast on that identification due to abundance constraints (e.g., Zhang et al. 2009). Sloan et al. (2014) examined these and other concerns about $\mathrm{MgS}$ and concluded that it remains a strong candidate for the carrier of the feature. Section 5.4 investigates this issue, but in the meantime, we will refer to this feature as the MgS feature for convenience.

We excluded objects if their spectra peak at wavelengths above $\sim 20 \mu \mathrm{m}$ (in $F_{\nu}$ units), because for carbon-rich sources, that indicates that they have evolved past the AGB. These excluded objects are part of the carbon-rich post-AGB sample studied by Sloan et al. (2014).

Two other classes of post-AGB objects have warmer spectral energy distributions (SEDs) peaking shortward of $\sim 20 \mu \mathrm{m}$ and are also excluded. $\mathrm{R} \mathrm{CrB}$ stars have smooth spectra showing only emission from amorphous carbon dust and no other significant spectral features. Kraemer et al. (2005) examined two in the SMC, but the IRS sample contains additional $\mathrm{R} \mathrm{CrB}$ stars (G. C. Clayton et al. 2016, in preparation). The other class of warm post-AGB objects includes two sources showing absorption bands from aliphatic hydrocarbons ${ }^{16}$ : SMP LMC 011 (Bernard-Salas et al. 2006), and MSX SMC 029 (Kraemer et al. 2006).

A variety of Spitzer observing programs contributed to the present sample of carbon stars (see Table 2). Each program comes with its own selection criteria and biases, which means that the present sample is neither uniform nor complete. However, thanks to the efforts of the designers of all the previous programs, the present sample probes carbon stars spanning a wide range of luminosities, colors, and evolutionary stages.

The first two programs listed in Table 2 were developed prior to launch as part of the guaranteed-time observations. Both sampled a variety of evolved stars, with Program 200 focused on supergiants and AGB stars identified as variable stars in the optical and near-IR in both the SMC and LMC. Program 1094 covered a range of post-main-sequence evolutionary stages in the LMC (and the Galaxy).

The next four programs were Guest Observer (GO) programs in Cycle 2 of the mission. Program 3277 started with the mid-

\footnotetext{
$\overline{16}$ I.e., non-aromatic species such as $\mathrm{C}_{2} \mathrm{H}_{6}$, longer chains, and other similar molecules.
} 
Table 2

Spectroscopic Spitzer Programs that Observed Magellanic Carbon Stars

\begin{tabular}{|c|c|c|}
\hline Program ID & Project Leaders & Description \\
\hline 200 & J. R. Houck \& G. C. Sloan & Evolved stars in the LMC and SMC \\
\hline 1094 & F. Kemper & AGB evolution in the LMC (and Galaxy) \\
\hline 3277 & M. P. Egan \& G. C. Sloan & $M S X$-based sample in the SMC \\
\hline 3426 & J. H. Kastner \& C. L. Buchanan & Bright infrared sources in the LMC \\
\hline 3505 & P. R. Wood \& A. A. Zijlstra & AGB stars in the LMC and SMC \\
\hline 3591 & F. Kemper & AGB evolution in the LMC \\
\hline 37088 & R. Sahai & Embedded carbon stars in the LMC \\
\hline 40650 & L. W. Looney \& R. A. Gruendl & YSOs and red sources in the LMC \\
\hline 40519 & A. G. G. M. Tielens \& F. Kemper & Extended the IRS sample in the LMC \\
\hline 50240 & G. C. Sloan \& K. E. Kraemer & Extended the IRS sample in the SMC \\
\hline 50338 & M. Matsuura & Carbon-rich post-AGB candidates in the LMC \\
\hline
\end{tabular}

IR sources in the SMC identified by the Mid-course Space Experiment (MSX; Price et al. 2001) to generate a sample spanning color-color space in the near- and mid-IR. Program 3426 sampled a randomly chosen subset of the brightest 150 sources in the LMC. Program 3505 identified AGB stars in several previous infrared surveys with the help of near-IR color-magnitude diagrams (CMDs). It covered both the LMC and SMC. Program 3591 extended Program 1094 to a larger sample in the LMC.

More programs followed later in the Spitzer mission. Program 37088 concentrated on heavily embedded and thus more evolved stars in the LMC. Program 40650 targeted about 300 candidate young stellar objects (YSOs) in the LMC, as well as several sources described as extremely red objects (EROs) and subsequently identified as deeply embedded carbon stars (Gruendl et al. 2008). The latter group is included in our sample. Program 40519 expanded on all previous programs studying the LMC by observing about 200 targets covering undersampled regions of color-color and colormagnitude space. It included evolved stars and YSOs, relied on the mid-IR photometry from the SAGE survey of the LMC (Surveying the Agents of Galactic Evolution; Meixner et al. 2006), and is known as SAGE-Spec. This program added a number of faint carbon-rich targets to the sample. Program 50240 expanded the targets observed in the SMC in a similar manner. Finally, Program 50338 concentrated on postAGB candidates in the LMC.

\subsection{Adopted Distances}

The known distances to the LMC and SMC enable us to study the total luminosities of the stars in our sample. We adopt distance moduli for the LMC and SMC of 18.5 and 18.9, respectively. Measurements of the distance modulus to the LMC cluster around our adopted value. The review by Feast (2013) leads to 18.50; another good example of a recent result is $18.49 \pm 0.05$ (Pietrzyński et al. 2013). Similarly, our adopted distance modulus to the SMC is close to the mean value reported by Rubele et al. (2015), $18.91 \pm 0.02$. The distances to these galaxies are only important to our determinations of absolute bolometric magnitudes, and because these have uncertainties larger than $0.1 \mathrm{mag}$, we are not concerned with precision in the distance modulus to further significant figures.

The SMC has a complicated structure, with considerable depth along the line of sight (Gardiner \& Hawkins 1991). Most of the stars reside in the Bar region, where the mean distance modulus to different regions can vary from $\sim 18.85$ to 19.02
(Rubele et al. 2015). Typical depths in this region are only $2-3 \mathrm{kpc}(\Delta(m-M) \lesssim 0.1)$, but in the extended Wing region to the east, Nidever et al. (2013) report depths up to $\sim 20 \mathrm{kpc}$ $(\Delta(m-M) \lesssim 0.8)$. Rubele et al. (2015) find mean distance moduli in the eastern regions between $\sim 18.65$ and 18.8. Most of the targets are in the Bar, and we will assume they are at our canonical distance modulus (18.9).

We have several targets in the cluster NGC 419, which is in the direction of the SMC. Glatt et al. (2008) give a distance modulus of 18.50, the same as for the LMC and about $10 \mathrm{kpc}$ in front of the SMC. They describe how the model which best fits the optical CMD gives $(m-M)_{o}=18.75$, while other models give values of 18.60 and 18.94 . It is unclear what justifies the reported value of 18.50 , and as discussed below, a value of 18.90 is more consistent with the IR data (see Section 4.3).

\subsection{IRS Observations}

The IRS observed the majority of the sources in the standard low-resolution nod sequence, most of them with both the ShortLow (SL) and Long-Low (LL) modules. SL and LL provide spectral coverage at 5-14 $\mu \mathrm{m}$ and $14-37 \mu \mathrm{m}$, respectively, with a resolution $(\lambda / \Delta \lambda)$ of $\sim 80-120$. Some of the fainter sources were observed only in SL. Generally, sources were observed in each of the two SL apertures, providing first- and second-order spectra, then the two LL apertures. Each source was observed in two nod positions in each aperture. Thus a full lowresolution spectrum requires eight pointings.

The carbon-rich EROs observed by Gruendl et al. (2008) used the SL module, along with Short-High (SH) and LongHigh (LH), which cover 10-19 and 19-36 $\mu \mathrm{m}$, respectively, at a resolution of $\sim 300$.

The reduction and calibration of the low-resolution spectra followed the standard Cornell algorithm (see Sloan et al. 2015a, for a more detailed description). The Cornell processing begins with the flatfielded images provided by version S18.18 of the pipeline at the Spitzer Science Center. Bad pixels in each image are identified and replaced with values based on their neighbors. All images for a given nod position are averaged, then a suitable background image is subtracted from the target image. In SL, the default is an aperture differerence, where the background for a given target is the image with the target in the other aperture, while in LL, the default is a nod difference, with the target in the same aperture, but the other nod. In some cases, the design of the observation or structure or other sources in the background forced a change from the default. 
We deviated from the reduction sequence described by Sloan et al. (2015a) by using an optimal-extraction algorithm to extract spectra from the images (Lebouteiller et al. 2010). This method fits a supersampled point-spread function (PSF) to the spatial profile in each row of the spectral image and can improve the signal-to-noise ratio $(\mathrm{S} / \mathrm{N})$ up to $80 \%$. It proved essential to generating usable spectra for the faintest sources in our sample. However, the method only works for point sources. For extended or mispointed objects, we used the default extraction algorithm which simply sums data in each wavelength element with no weighting.

Our pipeline continues with the standard step of combining spectra from the two nods and comparing the data to identify and reject spikes or divots which had survived the cleaning process. Spectral segments are then stitched together, shifting spectra upwards multiplicatively to the presumably bestcentered segment. Finally, extraneous data are removed from the ends of each segment.

Several previous papers on Magellanic carbon stars used the above algorithm, but without the optimal-extraction method which came later (Sloan et al. 2006; Zijlstra et al. 2006; Lagadec et al. 2007; Leisenring et al. 2008; Sloan et al. 2008; Kemper et al. 2010). The optimal extraction has generally improved the quality of the spectra.

\subsection{Photometric Data}

For all of our targets, we have constructed SEDs based on multi-epoch photometry in the optical, near-IR, and mid-IR.

The mid-IR data come from the SAGE survey of the LMC (Meixner et al. 2006) and the SAGE-SMC survey for the SMC (Gordon et al. 2011), which incorporates the $\mathrm{S}^{3} \mathrm{MC}$ survey (Spitzer Survey of the SMC; Bolatto et al. 2007). The SAGE surveys give two epochs, spaced approximately three months apart, for the Infrared Array Camera (IRAC) and the MultiImaging Photometer for Spitzer (MIPS). The $\mathrm{S}^{3} \mathrm{MC}$ survey provides one additional epoch in the core of the SMC. We use all four IRAC filters at 3.6, 4.5, 5.8, and $8.0 \mu \mathrm{m}$ and the MIPS $24 \mu \mathrm{m}$ filter. The SAGE-VAR survey adds four epochs from the Warm Spitzer Mission at 3.6 and $4.5 \mu \mathrm{m}$ for portions of the LMC and SMC (Riebel et al. 2015). We found matches with the SAGE-VAR survey for 61 of our carbon stars in the LMC and 26 in the SMC.

We also used additional epochs at 3.4 and $4.6 \mu \mathrm{m}$ from the Wide-field Infrared Survey Experiment (WISE; Wright et al. 2010) and the NEOWISE reactivation mission (Mainzer et al. 2014). ${ }^{17}$ For all WISE data, we utilized the multi-epoch data tables and collapsed them to one epoch approximately every six months. The publicly available data typically give us four epochs, two in 2010 and two in 2014.

Applying the color corrections derived for carbon stars in Appendix A allows us to convert $W 1(3.4 \mu \mathrm{m})$ to [3.6] and $W 2$ $(4.6 \mu \mathrm{m})$ to [4.5]. The combination of the SAGE surveys, including SAGE-VAR, and the WISE data can give us 10 or more epochs at 3.6 and $4.5 \mu \mathrm{m}$ for some of the stars in our sample.

The WISE survey is less sensitive and has lower angular resolution than the SAGE data, forcing us to reject some questionable data. We rejected both $W 1$ and $W 2$ for NGC 419

\footnotetext{
17 We used the AllWISE Multiepoch Photometry Table and the NEOWISE-R Single Exposure Source Table available online at the Infrared Science Archive (IRSA)
}

LE 35 and MIR 1 due to crowding. For IRAS 05026 and IRAS 05042, the multi-epoch data indicated a detection at W1, but the WISE images of the field showed nothing, even though the sources could be seen in $W 2$. The $W 1-W 2$ colors were inconsistent with the SAGE data, so we rejected the $W 1$ data but kept $W 2$.

Near-IR photometry comes from the 2MASS survey, and the deeper 2MASS-6X survey provides a second epoch at $J, H$, and $K_{s}$ (Cutri et al. 2012; Skrutskie et al. 2006). Additional epochs come from the Deep Near-IR Survey of the Southern Sky (DENIS) at $J$ and $K_{s}$ (Cioni et al. 2000) and the IR Survey Facility (IRSF) at $J, H$, and $K_{s}$ (Kato et al. 2007).

In the optical, we relied on the Magellanic Clouds Photometric Survey (MCPS) at $U, B, V$, and $I$ (Zaritsky et al. 2002, 2004). DENIS adds data at $I$. Additional mean magnitudes at $V$ and $I$ in the LMC come from the OGLE-III Shallow Survey (Ulaczyk et al. 2013). Where possible, we replaced the $V$ and $I$ data with mean magnitudes from the OGLE-III surveys of the Magellanic Clouds, which also give pulsation periods and amplitudes (Soszyński et al. 2009, 2011).

We searched for matches to the IRS sources first with the SAGE surveys, using positions estimated from the pointing of the IRS. The optimal extraction algorithm estimates the position of a source along a given slit (i.e., in the crossdispersion direction). For most observations, the nearly perpendicular orientation of the SL and LL slits helps to constrain the position of the source, despite the widths of the slits ( 3 " 6 and 10 " 6 , respectively). Where possible, we used matches to IRAC to update the initial IRS-based coordinates, then searched for 2MASS counterparts, again updating positions where possible, and then searched the other catalogs. The positions in Table 1 are the result.

The median offset from the estimated IRS position to IRAC is $0 . " 37$, consistent with a typical IRS pointing error of 0 ". 4 . The median offset between the IRAC and 2MASS positions is 0 ". 20. Comparing positions in the IRAC and MIPS catalogs, we find a median offset of 0 ". 20 .

For the most embedded sources, the expected photometry in the near-IR and at $3.6 \mu \mathrm{m}$ is near or below the detection limit, making it possible to mismatch the photometry to blue sources close to the expected positions of our carbon stars. We examined the environments of the most embedded sources, searching for targets within $1^{\prime}$ to estimate the source density. For IRAC, the source densities are 1.1-4.8 $\times 10^{-3}$, and for 2MASS-6X, they are higher, 7.0-8.2 $\times 10^{-3}$. Our maximum search radius in the LMC was 1 !" 25 , which gives a search field of 4.9 square arcsec and makes the odds of a mismatch for a given source $0.5 \%-2.4 \%$. For $2 \mathrm{MASS}-6 \mathrm{X}$, the odds of a mismatch are $3.4 \%-4.0 \%$ for a given source. Thus, mismatches are unlikely.

However, the above calculations were for field stars. For stars in clusters, we compared our near-IR results to the photometry reported by van Loon et al. (2005), and we used these data to replace the $J$ magnitude of one source, NGC 1978 MIR 1.

Tables 3 and 4 present the resulting photometry. For each photometric filter, the magnitude is a mean magnitude, and the uncertainty is the standard deviation of the data. When only one epoch of data in a given filter was available, we left the reported uncertainty undefined. While we can have over ten epochs at 3.6 and $4.5 \mu \mathrm{m}$, at longer wavelengths we are limited 
Table 3

Optical and Near-infrared Photometry

\begin{tabular}{|c|c|c|c|c|c|c|c|}
\hline Target & $\begin{array}{c}U \\
(\mathrm{mag})\end{array}$ & $\begin{array}{c}B \\
(\mathrm{mag})\end{array}$ & $\begin{array}{c}V \\
(\mathrm{mag})\end{array}$ & $\begin{array}{c}I \\
(\mathrm{mag})\end{array}$ & $\begin{array}{c}J \\
(\mathrm{mag})\end{array}$ & $\begin{array}{c}H \\
(\mathrm{mag})\end{array}$ & $\begin{array}{c}K \\
(\mathrm{mag})\end{array}$ \\
\hline GM 780 & $\ldots$ & $\ldots$ & $16.760 \pm \ldots$ & $14.235 \pm 0.040$ & $12.098 \pm 0.740$ & $10.658 \pm 0.564$ & $9.934 \pm 0.402$ \\
\hline MSX SMC 091 & $\ldots$ & $\ldots$ & $\ldots$ & $15.781 \pm 0.060$ & $13.764 \pm 0.374$ & $12.259 \pm 0.284$ & $10.829 \pm 0.191$ \\
\hline MSX SMC 062 & $\ldots$ & $\ldots$ & $\ldots$ & $16.985 \pm \ldots$ & $13.261 \pm 1.398$ & $11.418 \pm 0.159$ & $10.281 \pm 0.769$ \\
\hline MSX SMC 054 & $\ldots$ & $\ldots$ & $21.430 \pm \ldots$ & $20.383 \pm \ldots$ & $16.536 \pm 0.854$ & $14.449 \pm 0.182$ & $12.281 \pm 0.369$ \\
\hline 2MASS J004326 & $\ldots$ & $\ldots$ & $18.658 \pm \ldots$ & $15.170 \pm 0.040$ & $13.041 \pm 0.117$ & $11.857 \pm 0.108$ & $10.970 \pm 0.151$ \\
\hline MSX SMC 044 & $19.762 \pm 0.135$ & $19.230 \pm 0.039$ & $19.920 \pm 0.045$ & $16.406 \pm 1.882$ & $13.154 \pm 1.336$ & $12.178 \pm 1.822$ & $10.492 \pm 0.943$ \\
\hline MSX SMC 105 & $\ldots$ & $22.447 \pm 0.391$ & $21.099 \pm 0.303$ & $18.370 \pm 0.081$ & $15.151 \pm 0.446$ & $13.082 \pm 0.382$ & $11.245 \pm 0.290$ \\
\hline MSX SMC 036 & $\ldots$ & $\ldots$ & $\ldots$ & $19.462 \pm 0.090$ & $15.170 \pm 1.013$ & $13.553 \pm 0.898$ & $11.743 \pm 0.632$ \\
\hline GB S06 & $\ldots$ & $\ldots$ & $20.137 \pm \ldots$ & $18.114 \pm \ldots$ & $15.348 \pm 0.364$ & $13.146 \pm 0.283$ & $11.211 \pm 0.242$ \\
\hline MSX SMC 200 & $\ldots$ & $\ldots$ & $21.203 \pm \ldots$ & $17.557 \pm 0.200$ & $14.612 \pm 0.899$ & $12.724 \pm 0.804$ & $11.371 \pm 0.413$ \\
\hline
\end{tabular}

(This table is available in its entirety in machine-readable form.)

Table 4

Mid-infrared Photometry and Bolometric Magnitudes

\begin{tabular}{|c|c|c|c|c|c|c|}
\hline Target & $\begin{array}{c}{[3.6]} \\
\text { (mag) }\end{array}$ & $\begin{array}{c}{[4.5]} \\
(\mathrm{mag})\end{array}$ & $\begin{array}{c}{[5.8]} \\
(\mathrm{mag})\end{array}$ & $\begin{array}{c}{[8.0]} \\
(\mathrm{mag})\end{array}$ & $\begin{array}{c}{[24]} \\
(\mathrm{mag})\end{array}$ & $\begin{array}{c}M_{\mathrm{bol}} \\
(\mathrm{mag})\end{array}$ \\
\hline GM 780 & $8.738 \pm 0.454$ & $8.288 \pm 0.413$ & $8.283 \pm 0.064$ & $7.916 \pm 0.066$ & $6.771 \pm 0.368$ & -5.90 \\
\hline MSX SMC 091 & $9.602 \pm 0.366$ & $8.976 \pm 0.322$ & $8.260 \pm 0.008$ & $7.864 \pm 0.029$ & $7.328 \pm 0.060$ & -4.88 \\
\hline MSX SMC 062 & $8.941 \pm 0.266$ & $8.375 \pm 0.304$ & $7.639 \pm 0.026$ & $7.092 \pm 0.023$ & $6.272 \pm 0.178$ & -5.50 \\
\hline MSX SMC 054 & $9.940 \pm 0.327$ & $9.037 \pm 0.313$ & $8.408 \pm 0.201$ & $7.771 \pm 0.142$ & $6.595 \pm 0.142$ & -4.58 \\
\hline 2MASS J004326 & $10.495 \pm 0.400$ & $10.476 \pm 0.257$ & $10.196 \pm 0.095$ & $9.615 \pm 0.061$ & $9.316 \pm 0.056$ & -4.56 \\
\hline MSX SMC 044 & $9.300 \pm 0.256$ & $8.661 \pm 0.230$ & $8.189 \pm 0.191$ & $7.713 \pm 0.155$ & $7.216 \pm 0.077$ & -5.29 \\
\hline MSX SMC 105 & $9.312 \pm 0.129$ & $8.544 \pm 0.151$ & $8.007 \pm 0.079$ & $7.370 \pm 0.078$ & $5.928 \pm 0.220$ & -5.04 \\
\hline MSX SMC 036 & $9.698 \pm 0.314$ & $8.790 \pm 0.274$ & $8.051 \pm 0.199$ & $7.515 \pm 0.160$ & $6.529 \pm 0.131$ & -4.62 \\
\hline GB S06 & $8.666 \pm 0.259$ & $7.927 \pm 0.373$ & $6.922 \pm 0.157$ & $6.241 \pm 0.182$ & $4.874 \pm 0.098$ & -5.62 \\
\hline MSX SMC 200 & $9.298 \pm 0.180$ & $8.806 \pm 0.198$ & $8.362 \pm 0.062$ & $7.894 \pm 0.030$ & $7.276 \pm 0.206$ & -4.81 \\
\hline
\end{tabular}

(This table is available in its entirety in machine-readable form.)

to three epochs in the heart of the SMC and two epochs in the LMC and the outskirts of the SMC.

\subsection{Galactic Comparison Spectra}

We also consider a Galactic control sample using spectra from the Short-Wavelength Spectrometer (SWS; de Graauw et al. 1996) on the Infrared Space Observatory (ISO; Kessler et al. 1996). Leisenring et al. (2008) presented the original list of 34 carbon stars used in earlier comparisons to Magellanic samples. The present sample includes eight additional objects, for a total of 42 .

The comparison SWS sample was chosen from spectra classified as carbon stars by Kraemer et al. (2002). Their classification scheme assigns spectra to groups based on the overall shape of the spectrum, with naked stars in Group 1, stellar spectra showing some dust emission in Group 2, spectra dominated by warm dust in Group 3, and spectra dominated by cold dust (so that the spectrum peaks past $\sim 20 \mu \mathrm{m}$ ) in Groups 4 and 5. Spectra are assigned to subgroups based on the dominant features in the spectra. The carbon stars are those with the following classifications: 1.NC (naked star with carbon-rich molecular absorption bands), 2.CE and 3.CE (optically thin carbon-rich dust emission), and 3.CR (reddened spectra from optically thick carbon-rich dust emission). The original sample of 34 described by Leisenring et al. (2008) did not include any naked carbon stars; we have added three 1.NC sources. Nor did it consider the 23 slower SWS scans, which adds three more sources. The additional two sources were simply overlooked before.

\section{ANALYSIS}

\subsection{The Manchester Method}

Sloan et al. (2006) and Zijlstra et al. (2006) introduced the Manchester method to extract and compare information uniformly from large samples of infrared spectra from carbon stars. The key metric is the [6.4]-[9.3] color, which samples the spectrum at two wavelengths that are relatively free of molecular absorption bands and solid-state emission features. This color reddens as the amount of amorphous carbon grows above the stellar photosphere. Groenewegen et al. (2007) found that the [6.4]-[9.3] color increased linearly with the log of the dust-production rate (DPR). The current sample includes bluer sources which deviate from a linear relationship, and it requires a more complex formulation (e.g., Matsuura et al. 2009; Gullieuszik et al. 2012; Riebel et al. 2015). All of these formulations are based on a tight relationship between color and DPR and reinforce our assumption that the [6.4]-[9.3] color is a good proxy for DPR.

The Manchester method measures the equivalent width of the acetylene absorption bands at 7.5 and $13.7 \mu \mathrm{m}$ by fitting line segments to the continua to either side. The $13.7 \mu \mathrm{m}$ band is actually just the Q branch of a broader feature, and we will focus on the stronger $7.5 \mu \mathrm{m}$ band. The strength of the $\mathrm{SiC}$ dust emission feature at $\sim 11.5 \mu \mathrm{m}$ is measured similarly and is 


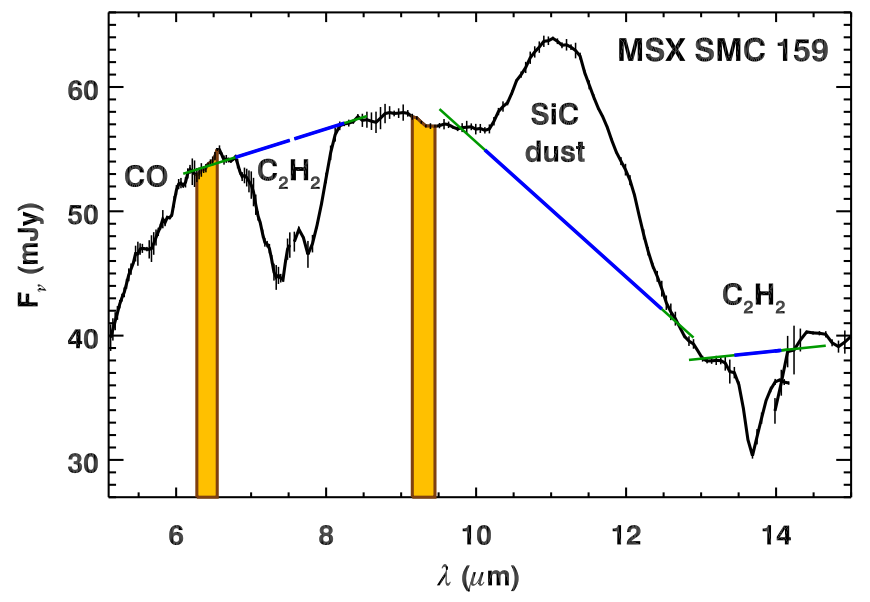

Figure 1. Manchester method applied to the 5-15 $\mu \mathrm{m}$ spectrum of MSX SMC 159. The [6.4]-[9.3] color provides an estimate of the relative contributions of stellar photosphere and amorphous carbon dust in two spectral regions relatively free of absorption or emission features. Line segments are used to estimate the continuum under or over the $\mathrm{C}_{2} \mathrm{H}_{2}$ absorption band at $7.5 \mu \mathrm{m}$, the $\mathrm{Q}$ branch of the $\mathrm{C}_{2} \mathrm{H}_{2}$ band at $13.7 \mu \mathrm{m}$, and the $\mathrm{SiC}$ dust emission feature at $\sim 11.3 \mu \mathrm{m}$.

reported as a ratio of integrated flux in the feature to the integrated flux in the underlying continuum.

We also measure the strength of the $26-30 \mu \mathrm{m}$ feature attributed to $\mathrm{MgS}$, but because the IRS spectral coverage cuts off the long-wavelength side of the feature, we use a two-step process. First we estimate a continuum based on the apparent color of the spectrum at 16.5 and $21.5 \mu \mathrm{m}$. Then we integrate the spectrum above this estimated continuum. If the spectra do not appear to be turning down at the long-wavelength cut-off, we assume that the $26-30 \mu \mathrm{m}$ feature is not present or is contaminated in some way and do not report a value. As with the $\mathrm{SiC}$ feature, we report the integrated flux in the feature, divided by the integrated continuum.

Figures 1 and 2 illustrate the application of the Manchester method to the spectrum of one Magellanic carbon star, MSX SMC 159. Sloan et al. (2006) presented similar figures for the same star (see their Figures 4 and 5). The difference is that we used optimal extraction for the spectrum here, with a noticeable improvement in $\mathrm{S} / \mathrm{N}$.

Table 5 presents the results for the IRS sample of carbon stars in the Magellanic Clouds. Table 6 presents the 42 Galactic carbon stars observed with the SWS. The results in Table 6 differ from those presented by Leisenring et al. (2008) because of differences in how the continuum was estimated. As explained in Appendix A, the [6.4]-[9.3] colors of two sources, IRAS 04589 and IRAS 05306, were artificially reddened because the spectra were badly mispointed. For these, we replaced the [6.4]-[9.3] colors with values estimated from their photometric [5.8]-[8] color.

\subsection{Spectral Classification}

Table 5 also includes infrared spectral classifications, which are based on modifications to the two-level scheme applied by Kraemer et al. (2002) to the SWS data (see Section 2.5). The original scheme envisioned a third level of classification for some of the more populated subgroups, but this level was adopted only for the silicate emission (SE) sources by using the SE indices defined by Sloan \& Price (1995).

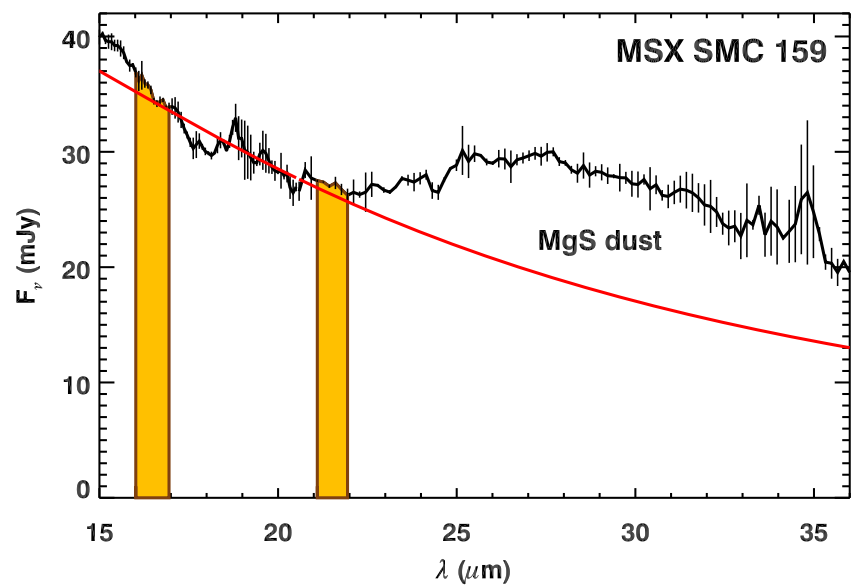

Figure 2. Manchester method applied to the 15-37 $\mu \mathrm{m}$ spectrum of MSX SMC 159. The [16.5]-[21.5] color estimates the continuum temperature at longer wavelengths in order to estimate the continuum under the $\mathrm{MgS}$ dust emission feature.

The scheme as modified here changes how carbon stars are classified. Previously, they fell into four groups in the sequence 1.NC-2.CE-3.CE-3.CR as the carbon-rich dust shell grew progressively thicker and redder. The new scheme replaces this sequence with CE0-CE5, depending solely on the [6.4]-[9.3] color, with the breaks at a color of 0.05 and at intervals of 0.30 from there to 1.25 .

Figure 3 uses the classifications of Galactic carbon stars by Kraemer et al. (2002) to show that the old classifications closely follow the [6.4]-[9.3] color. The new sequence shifts the boundaries between the subclasses, but presents them in a more easily recognizable sequence.

Some of the redder CE5 sources show negative values for the SiC-to-continuum ratio, because the $\mathrm{SiC}$ feature is in absorption instead of emission. For those sources with a $3 \sigma$ detection or better, we have designated them as "CA" instead of "CE," for absorption instead of emission. This classification is analogous to "SA" versus "SE" introduced for silicates by Sloan \& Price (1995).

\section{RESULTS}

The present sample has added over 70 sources to the previously published samples of Magellanic carbon stars. The first question to address is whether or not the larger sample contradicts any earlier conclusions. We therefore base our analysis, as before, on comparisons using the [6.4]-[9.3] color as a proxy for DPR. This approach compares the properties of the stars and their spectra from populations with different metallicities but similar DPRs.

\subsection{The [6.4]-[9.3] Color}

Figure 4 shows how the [6.4]-[9.3] color of the carbon stars depends on pulsation period. The larger sample considered here reinforces the previous conclusions. First, the DPR, as measured by the [6.4]-[9.3] color, generally increases with longer pulsation periods, once the period exceeds $\sim 250$ days. Second, the scatter is significant, with a range of DPRs possible at a given pulsation period. Third, the three galaxies considered show no obvious differences, indicating that metallicity does not have a noticeable effect. 
Table 5

Spectroscopic Data-IRS Sample

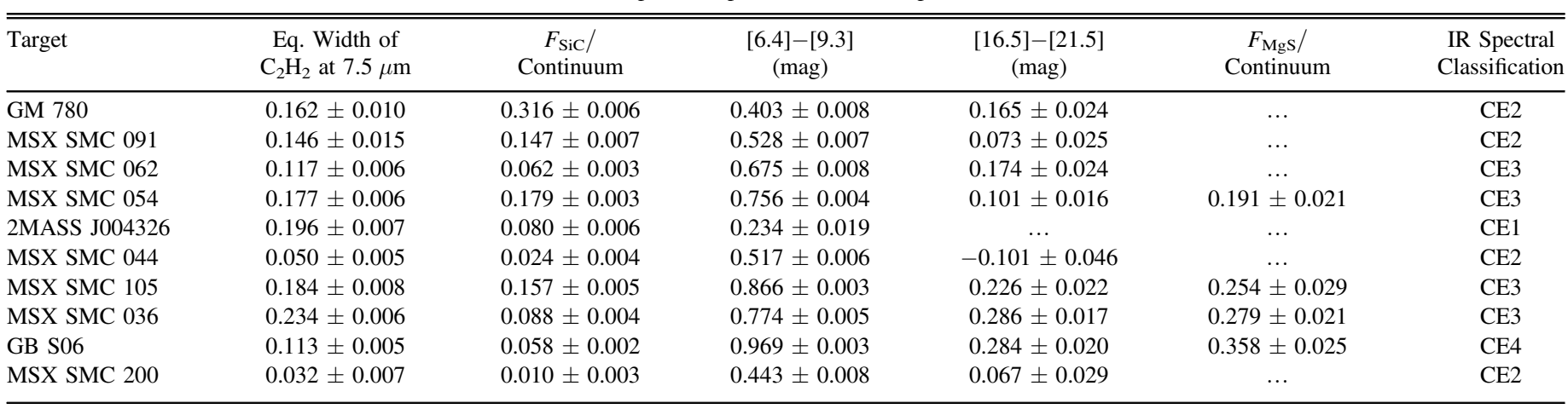

(This table is available in its entirety in machine-readable form.)

Table 6

Spectroscopic Data-SWS Sample

\begin{tabular}{|c|c|c|c|c|c|c|c|}
\hline Target & Alias & $\begin{array}{c}\text { Eq. Width of } \\
\mathrm{C}_{2} \mathrm{H}_{2} \text { at } 7.5 \mu \mathrm{m}\end{array}$ & $\begin{array}{c}F_{\mathrm{SiC}} / \\
\text { Continuum }\end{array}$ & $\begin{array}{c}{[6.4]-[9.3]} \\
(\mathrm{mag})\end{array}$ & $\begin{array}{c}{[16.5]-[21.5]} \\
(\mathrm{mag})\end{array}$ & $\begin{array}{c}F_{\mathrm{MgS} /} / \\
\text { Continuum }\end{array}$ & $\begin{array}{l}\text { IR Spectral } \\
\text { Classification }\end{array}$ \\
\hline WZ Cas & $\ldots$ & $0.347 \pm 0.007$ & $-0.022 \pm 0.002$ & $0.029 \pm 0.002$ & $0.438 \pm 0.004$ & . & CE0 \\
\hline VX And & $\ldots$ & $0.238 \pm 0.001$ & $0.182 \pm 0.001$ & $0.123 \pm 0.003$ & $0.067 \pm 0.010$ & $\ldots$ & CE1 \\
\hline R Scl & $\ldots$ & $0.290 \pm 0.002$ & $0.205 \pm 0.001$ & $0.273 \pm 0.002$ & $0.266 \pm 0.002$ & $0.292 \pm 0.004$ & CE1 \\
\hline R For & $\ldots$ & $0.125 \pm 0.001$ & $0.279 \pm 0.001$ & $0.361 \pm 0.001$ & $0.131 \pm 0.003$ & $\ldots$ & CE2 \\
\hline AFGL 341 & V596 Per & $0.063 \pm 0.001$ & $0.073 \pm 0.001$ & $1.665 \pm 0.005$ & $0.407 \pm 0.008$ & $0.411 \pm 0.010$ & CE5 \\
\hline W Ori & $\ldots$ & $0.064 \pm 0.001$ & $0.229 \pm 0.001$ & $0.055 \pm 0.001$ & $-0.054 \pm 0.004$ & $0.137 \pm 0.002$ & CE1 \\
\hline IRC -10095 & V1187 Ori & $0.277 \pm 0.002$ & $0.192 \pm 0.001$ & $0.163 \pm 0.003$ & $0.196 \pm 0.008$ & $0.784 \pm 0.012$ & CE1 \\
\hline
\end{tabular}

(This table is available in its entirety in machine-readable form.)

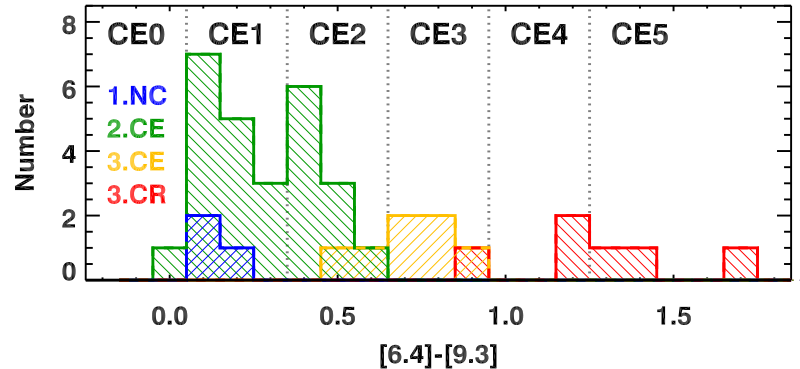

Figure 3. Distribution of the Galactic carbon stars in our control sample with [6.4]-[9.3] color, segregated by their infrared spectral classifications from Kraemer et al. (2002). The [6.4]-[9.3] color increases nearly monotonically along the sequence 1.NC-2.CE-3.CE-3.CR.

\subsection{Infrared Spectral Features}

\subsubsection{Silicon Carbide Dust}

Figure 5 shows that the dependence of the strength of the $\mathrm{SiC}$ feature on the [6.4]- [9.3] color changes with host galaxy. For the Galactic sources, the $\mathrm{SiC}$ dust emission increases quickly from [6.4] $-[9.3]=0$ to $\sim 0.4$, then drops steadily to a color of $\sim 1.7$, which is the maximum in the Galactic sample. Most of the carbon stars in the SMC sample behave differently, with $\mathrm{SiC}$ strength rising more gradually with color to [6.4] $-[9.3] \sim 1.4$ for the reddest carbon star observed with the IRS in the SMC. The LMC sample is more evenly split between the upper and lower sequences.

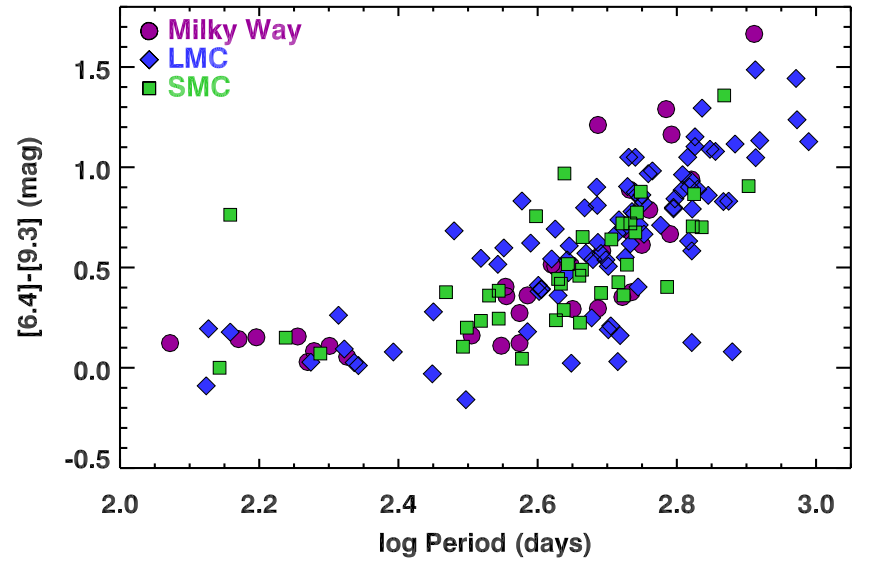

Figure 4. [6.4]-[9.3] color of the carbon stars in the Galaxy, LMC, and SMC as a function of pulsation period. For periods $\gtrsim 250$ days, the colors generally grow redder with longer periods, but with considerable scatter. No dependency on metallicity is apparent.

Sloan et al. (2006) first noticed this metallicity-dependent difference when they compared samples from the Galaxy and the SMC. As more carbon stars were added from the SMC (Lagadec et al. 2007) and LMC (Zijlstra et al. 2006; Leisenring et al. 2008), the two tracks in Figure 5 remained separate. Zijlstra et al. (2006) pointed out that metallicity will determine the availability of silicon, so that metal-rich stars generally populate the sequence with stronger $\mathrm{SiC}$ emission while metalpoor stars show weaker $\mathrm{SiC}$ features. 


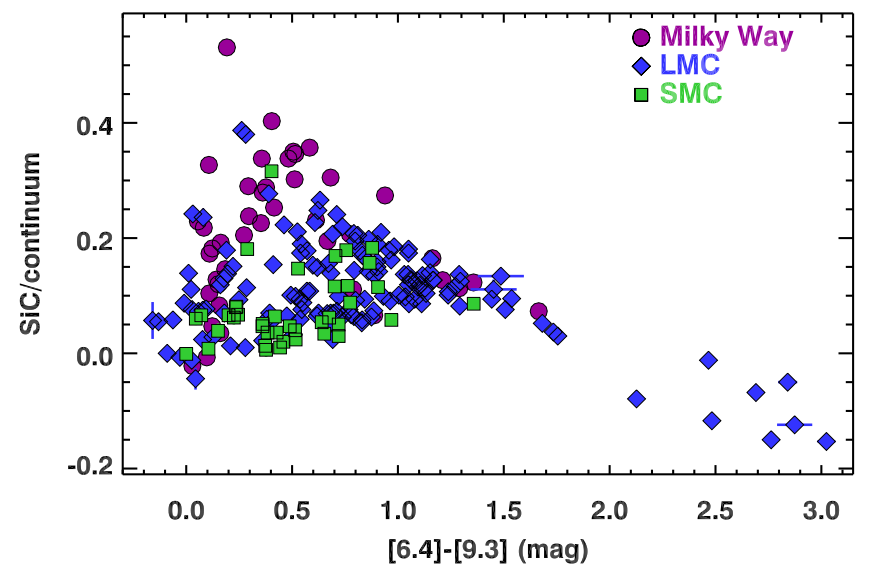

Figure 5. Strength of $\mathrm{SiC}$ dust emission as a function of [6.4]-[9.3] color. As the [6.4]-[9.3] color increases, stars follow one of two tracks. The SiC strength increases quickly in more metal-rich stars, then turns over and decreases. In metal-poor stars, the $\mathrm{SiC}$ strength increases more gradually. Most of the stars with [6.4]-[9.3] $\gtrsim 2.0$ have negative $\mathrm{SiC}$ strengths, indicating absorption.

All eight of the stars with [6.4]-[9.3] $\gtrsim 2.0$ have negative $\mathrm{SiC} /$ continuum ratios, which indicate $\mathrm{SiC}$ absorption in optically thick dust shells. The seven carbon-rich EROs observed by Gruendl et al. (2008) all show SiC absorption; two of these were re-observed as potential post-AGB objects by Matsuura et al. (2014). The eighth source was part of the SAGE-Spec program (Kemper et al. 2010). All have spectroscopic properties consistent with deeply embedded objects near the end of their AGB lifetimes.

\subsubsection{Magnesium Sulfide Dust}

Figure 6 shows how the MgS emission increases with [6.4] - [9.3] in the different populations. $\mathrm{MgS}$ is apparent in the Galactic sample at all [6.4]-[9.3] colors, while in the LMC, the first $\mathrm{MgS}$ feature is at [6.4]-[9.3] 0.25. No other LMC source shows $\mathrm{MgS}$ until [6.4]-[9.3] reaches a value $\sim 0.5$. In the SMC, MgS does not appear until [6.4]-[9.3] 0.7.

Figure 7 illustrates the behavior of the $\mathrm{MgS}$ emission differently, by plotting the mean $\mathrm{MgS} /$ continuum strength for all data up to a given [6.4]-[9.3] color. It shows more clearly how the LMC lags the Milky Way, with the SMC even further behind, as the [6.4]-[9.3] color increases. The traces for the Milky Way and SMC are unchanged past colors of $\sim 1.6$ and 1.4 , respectively, due to a lack of redder sources. The lower abundances of $\mathrm{Mg}$ and $\mathrm{S}$ in more metal-poor stars appear to be delaying the condensation of MgS until the DPR is higher and the dust is cooler.

\subsubsection{Acetylene Gas at $7.5 \mu \mathrm{m}$}

Figure 8 compares the behavior of the $7.5 \mu \mathrm{m}$ absorption band from $\mathrm{C}_{2} \mathrm{H}_{2}$ for the different samples. For the less dusty stars $([6.4]-[9.3] \lesssim 0.35)$, all three galaxies show a range of band strengths, but to the red, the upper bound diminishes. Figure 9 clarifies the behavior of each galaxy by plotting the mean equivalent width and color for each CE class. For CE2 and redder sources, the mean equivalent width of the $7.5 \mu \mathrm{m}$ acetylene band is consistently strongest in the SMC and weakest in the Galaxy.

As shown below (Section 4.5), all of the CE0 and most of the $\mathrm{CE} 1$ sources are associated with relatively dust-free carbon

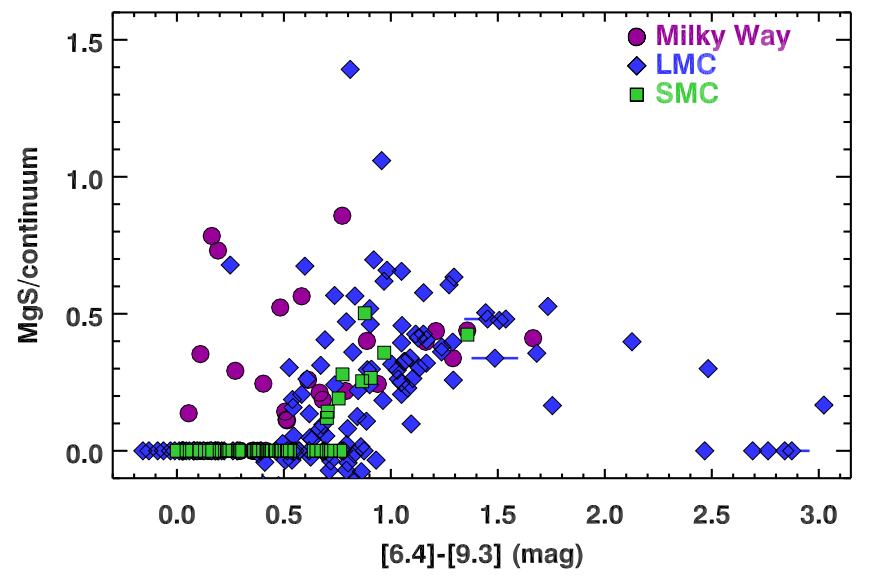

Figure 6. Strength of $\mathrm{MgS}$ dust emission as a function of [6.4]-[9.3] color. More metal-poor stars have to have redder [6.4]-[9.3] colors before $\mathrm{MgS}$ becomes visible.

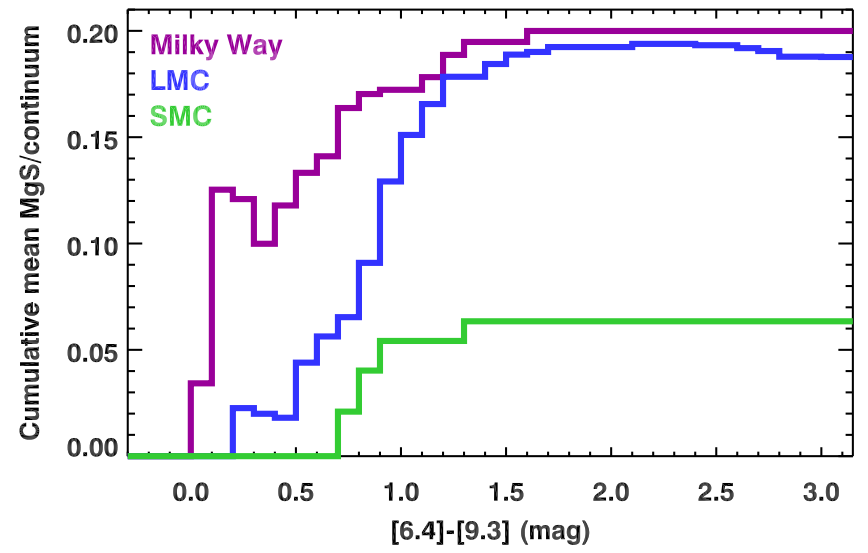

Figure 7. Cumulative mean $\mathrm{MgS}$ emission. At each color, the mean $\mathrm{MgS} /$ continuum strength for all sources with bluer colors is plotted.

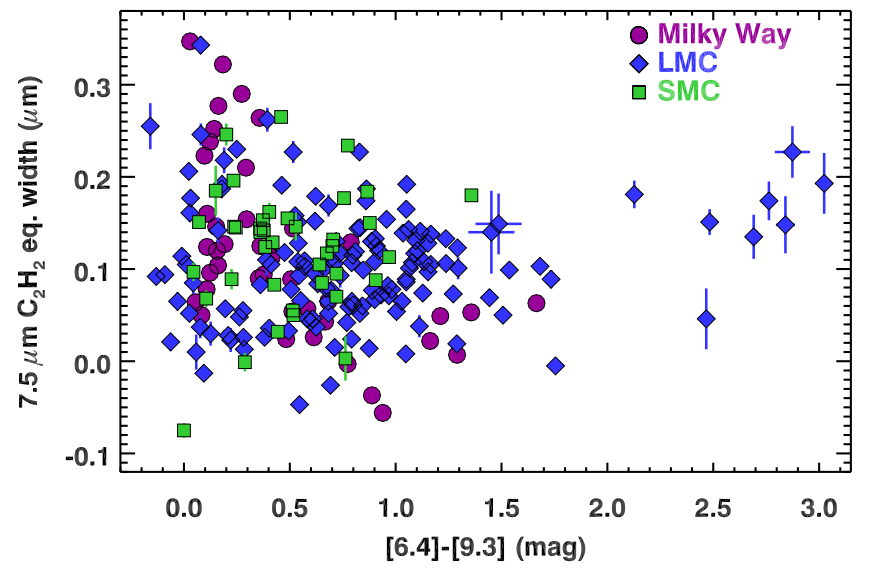

Figure 8. Strength of the $7.5 \mu \mathrm{m} \mathrm{C}_{2} \mathrm{H}_{2}$ absorption band vs. [6.4]-[9.3] color.

stars which can have strong molecular absorption bands due to the lack of veiling from the dust surrounding the redder sources. Sloan et al. (2015b) found that in the SMC, the dusty stars with veiled molecular bands usually are pulsating in the fundamental mode with strong amplitudes as Mira variables, while the other group are detected as semi-regulars or irregulars. To focus on the dust-production process, we should focus on the stars classified as CE2 or later. 


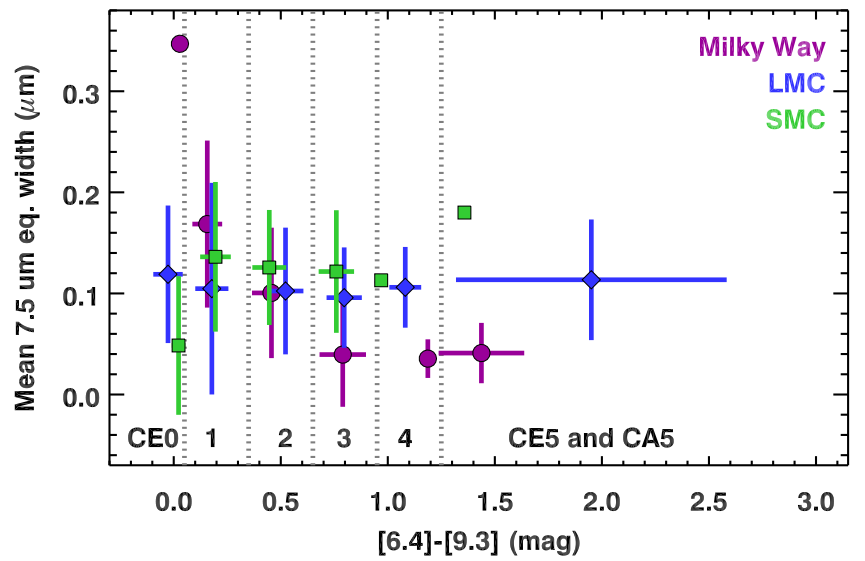

Figure 9. Strength of the $7.5 \mu \mathrm{m} \mathrm{C}_{2} \mathrm{H}_{2}$ absorption band vs. [6.4]-[9.3] color, binned by $\mathrm{CE}$ class to illustrate the general trends in the data. When determining means, negative equivalent widths have been treated as zero, because acetylene emission is unlikely.

To make a quantitative comparison of the $7.5 \mu \mathrm{m}$ acetylene band, we examine the CE2-3 sources, because the CE4 and CE5 classes have only one SMC source each. We set negative equivalent widths to zero, because $7.5 \mu \mathrm{m}$ emission is unlikely. For the chosen color range, the mean equivalent width of the $7.5 \mu \mathrm{m}$ band decreases from $0.12 \pm 0.01 \mu \mathrm{m}$ in the SMC to $0.10 \pm 0.01 \mu \mathrm{m}$ in the LMC and to $0.08 \pm 0.02 \mu \mathrm{m}$ in the Galaxy (the quoted errors are the uncertainty in the mean). While the spread in each sample is substantial, the steady drop in mean band strength is still apparent. Thus the expanded IRS sample has not changed the conclusions drawn before about the $7.5 \mu \mathrm{m}$ acetylene band.

While the behaviors of the $\mathrm{C}_{2} \mathrm{H}_{2}$ absorption at $7.5 \mu \mathrm{m}$ and the $\mathrm{SiC}$ emission at $11.5 \mu \mathrm{m}$ differ quantitatively, their rough similarity raises a possible concern, which we have examined and ruled out. Acetylene also shows a strong absorption band centered at $13.7 \mu \mathrm{m}$, but the sharp absorption band apparent at $13.7 \mu \mathrm{m}$ is just from the $\mathrm{Q}$ branch $(\Delta J=0)$, with the $\mathrm{P}$ and $\mathrm{R}$ branches extending the feature over a micron in either direction. The possible SiC emission could be affected by strong $\mathrm{C}_{2} \mathrm{H}_{2}$ absorption to the red, which could push the continuum fit down and artifically add to the measured emission. We investigated by shifting the wavelengths used to fit the continuum on the red side of the $\mathrm{SiC}$ feature. Figure 1 shows an inflection on the SiC feature at $\sim 12.0 \mu \mathrm{m}$. We shifted the red continuum wavelengths to this inflection and recalculated the intregated $\mathrm{SiC}$ flux for our samples, and found that, while the total $\mathrm{SiC}$ emission certainly grew smaller, the samples behaved the same qualitatively, and the differences in how the three galaxies behaved were unchanged.

\subsection{Bolometric Magnitudes}

For each star in the Magellanic sample, we can estimate its bolometric magnitude $\left(M_{\text {bol }}\right)$ by integrating its SED, which is determined from the IRS spectra and the mean magnitudes from the multi-epoch photometry described in Section 2.4. We integrated through the photometry below $5 \mu \mathrm{m}$ using linear interpolation, then integrated the IRS spectrum. To the blue of the optical photometry, we assumed a Wien distribution. To the red of the IRS data, we assumed a Rayleigh-Jeans tail. If the IRS spectrum only included data from SL (i.e., stopped at

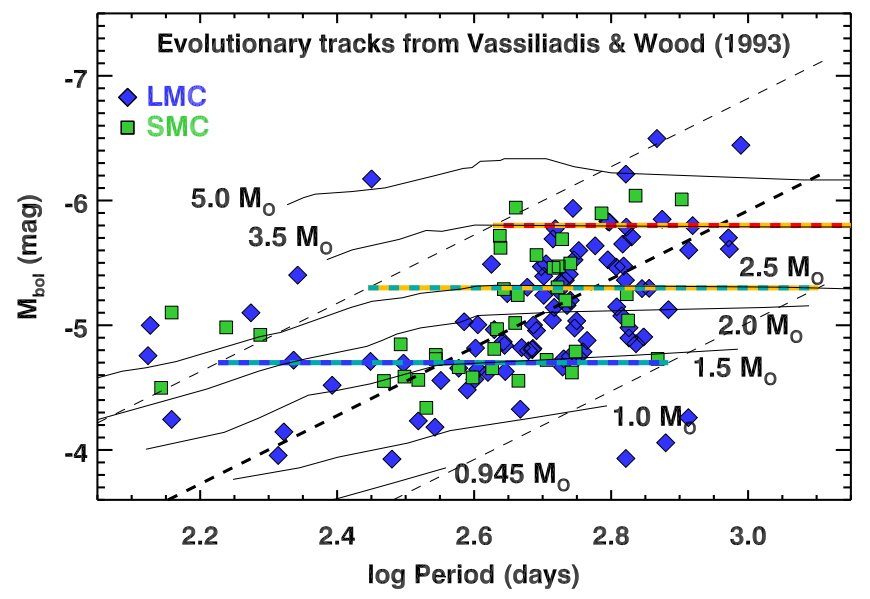

Figure 10. Period-luminosity plot showing how we binned the Magellanic carbon stars into four initial-mass groups based on their bolometric luminosities. The thick dashed line is the period-luminosity relation fitted to the fundamental-mode pulsators, and the thin dashed lines to either side enclose the range we will consider to be on the fundamental-mode sequence. Within this range, evolutionary tracks on the AGB are generally close to horizontal, as demonstrated by the tracks from Vassiliadis \& Wood (1993). The multicolored horizontal lines show the boundaries between the mass bins.

$14 \mu \mathrm{m})$, we also utilized the MIPS data at $24 \mu \mathrm{m}$. The resulting estimates for $M_{\mathrm{bol}}$ appear in the last column of Table 4.

Our IRS sample includes six carbon stars in NGC 419. At an assumed distance modulus of 18.90, the median absolute magnitude for these stars $=-4.79 \pm 0.23$, with $M_{\mathrm{bol}}=-5.24$ for NGC 419 IR 1 and -4.73 for NGC 419 MIR 1 . These values are consistent with an estimated zero-age main-sequence mass of $1.9 M_{\odot}$ (Kamath et al. 2010) and an age of $1.35 \mathrm{Gyr}$ (Girardi et al. 2009), based on models of the AGB and red clump, respectively. The bolometric magnitudes of NGC 419 compare well with the rest of the SMC, which has $\left\langle M_{\text {bol }}\right\rangle=-5.03 \pm 0.49$. If NGC 419 were in front of the SMC at a distance modulus of 18.50 (Glatt et al. 2008), then its median $M_{\mathrm{bol}}$ would lie outside the $1 \sigma$ range for the SMC, making it older than most of the carbon stars observed by the IRS in the SMC.

Figure 10 includes evolutionary tracks from Vassiliadis \& Wood (1993) on the period-luminosity plane and also shows the location of the $\mathrm{P}-\mathrm{L}$ relation for fundamental-mode pulsators. For stars with higher initial mass and for lowermass stars with longer periods, the evolutionary tracks are nearly horizontal, so that $M_{\mathrm{bol}}$ can be used as a proxy for initial mass. We have therefore divided the fundamental-mode pulsators into four mass bins with the horizontal lines defined in Figure 10 as boundaries. While the evolutionary tracks do deviate from horizontal for shorter pulsation periods, the figure shows that this has little impact on the stars in our sample, provided we only consider stars with periods $\gtrsim 250$ days $(\log P \sim 2.4)$.

It should be emphasized that the estimates for initial mass are rough and are not to be taken too literally or quantitatively. The key is the steady bolometric magnitude on the late stages of the evolutionary tracks. These make it possible to use $M_{\mathrm{bol}}$ to segregate the data by initial mass for statistical purposes.

The thick black dashed line in Figure 10 is a periodluminosity relation, first fitted to all of the Magellanic stars in our sample with periods $>250$ days, then fitted iteratively to only those within an envelope of \pm 0.9 mag to avoid stars not on the fundamental-mode sequence. The linear solution 


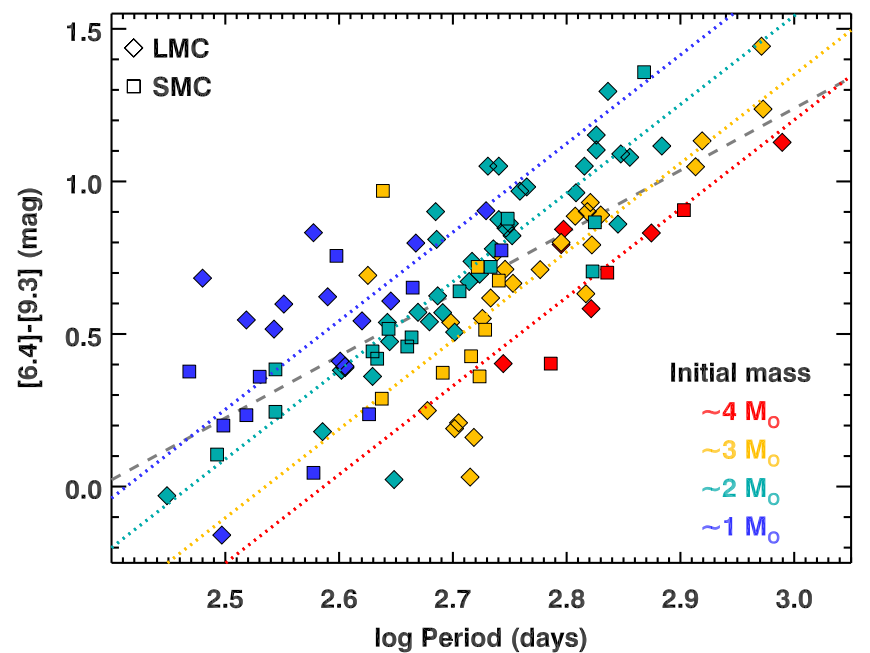

Figure 11. [6.4]-[9.3] color as a function of pulsation period with the Magellanic sample, color-coded by their initial mass as estimated from their bolometric magnitude. Sources not assigned to a mass bin are not included. The width of the relation between [6.4]-[9.3] color and period clearly arises from the mass distribution of the samples. The gray dashed line is a line fitted to all of the data, while the colored dotted lines are fitted to each mass bin separately (see Section 4.3).

converges quickly:

$$
M_{\mathrm{bol}}=2.30-2.74 \log P(\text { days }) .
$$

This line is steeper than the $\mathrm{P}-\mathrm{L}$ relation by Whitelock et al. (2006). They found a slope of -2.54 using bolometric corrections to estimate $M_{\text {bol }}$, while our estimates are based on integrations through the IRS data and the available photometry at shorter wavelengths. Sloan et al. (2015b) noted that the estimated bolometric magnitudes of the dustiest sources, which tend to have the longest periods, depend on the precise method used. Here, we have spectral data covering the peak of the SED for those sources, which should improve on the cases where only photometry is available.

\subsection{The Period-Color Relation and Initial Mass}

Figure 11 reprises Figure 4, except that the sources are colorcoded by our rough estimates of their initial mass. Figure 11 explains the width of the relationship of [6.4]-[9.3] versus period. The Magellanic samples include a range of masses, each of which follows its own narrow relation. When stars switch from overtone pulsations to the fundamental mode, they begin to produce dust at significant rates for the first time, and this rate increases as their pulsation period increases. The slope to the period-luminosity relation means that this mode switch occurs at longer pulsation periods for more luminous (and thus more massive) stars, which explains why segregating by mass reveals the parallel tracks in Figure 11. The finite width of the period-luminosity relation suggests that this scenario of increasing pulsation period and increasing DPR and mass-loss rate can be sustained for a limited time.

Sloan et al. (2008) concluded that for Magellanic carbon stars, metallicity did not play a detectable role in dust production, because the Galaxy, LMC, and SMC showed indistinguishable relations between [6.4]-[9.3] color and pulsation period. However, more metal-poor carbon stars in other nearby Local Group dwarf galaxies $([\mathrm{Fe} / \mathrm{H}] \lesssim-1)$ do
Table 7

Period-corrected Differences in [6.4]-[9.3] Color

\begin{tabular}{lcc}
\hline \hline \multirow{2}{*}{$\begin{array}{l}\text { Approximate } \\
\text { Initial Mass }\left(M_{\odot}\right)\end{array}$} & \multicolumn{2}{c}{$\Delta(\mathrm{LMC})-\Delta(\mathrm{SMC})(\mathrm{mag})^{\mathrm{a}}$} \\
\cline { 2 - 3 } & Using One Fitted Line & Fitting to Each Mass Bin \\
\hline$\sim 4$ & $0.083 \pm 0.098$ & $-0.001 \pm 0.077$ \\
$\sim 3$ & $0.147 \pm 0.100$ & $0.054 \pm 0.103$ \\
$\sim 2$ & $0.112 \pm 0.048$ & $0.032 \pm 0.043$ \\
$\sim 1$ & $0.157 \pm 0.093$ & $0.131 \pm 0.100$ \\
\hline
\end{tabular}

Note.

${ }^{\mathrm{a}} \Delta=\operatorname{median}\left\{([6.4]-[9.3])_{\mathrm{obs}}-([6.4]-[9.3])_{\text {line }}\right\}$.

show less dust than might be expected for their pulsation period (Sloan et al. 2012).

The data in Figure 11 point to the need to account for the different mass distributions of the carbon stars observed by the IRS in the LMC and SMC in order to re-assess if the role of metallicity can be detected for $[\mathrm{Fe} / \mathrm{H}] \gtrsim-1$. A line fitted to all of the data gives

$$
[6.4]-[9.3]=-4.52+1.91 \log P \text { (days). }
$$

For each star, we used this line to predict the expected [6.4] $-[9.3]$ color based on the pulsation period and compared it to the observed [6.4]-[9.3] color. For each mass bin and for each galaxy, we computed the median of these offsets. Table 7 gives the difference in these median offsets, along with the propagated uncertainty in the mean (in the column labeled "Using One Fitted Line"). Averaging the differences between LMC and SMC across the mass bins reveals a redder [6.4] $-[9.3]$ color in the LMC of $0.125 \pm 0.042 \mathrm{mag}$, which is a $2.9 \sigma$ result.

The data in each mass bin in Figure 11 follow a steeper slope than the total data set, and the carbon stars in the LMC and SMC do not have the same period distributions. To further correct for this difference in the sample, we have fitted lines independently to the four mass bins. Because these lines are driven somewhat by the noise in our data, we found the weighted mean of the slopes (2.91 mag/dex) and used this slope for each mass bin. The colored dotted lines in the figure illustrate the results. Table 7 gives the resulting median difference in [6.4]-[9.3] between the LMC and SMC. The average from the four mass bins is $0.054 \pm 0.040$, which is only a $1.3 \sigma$ result.

We conclude that the full sample of Magellanic carbon stars observed by the IRS shows hints of a subtle dependence of DPR with metallicity, but once we account for different distributions by initial mass and metallicity, our sample does not reveal a statistically significant result. The effects of pulsation period and initial mass on dust production are much stronger.

\subsection{Color-Color and Color-Magnitude Space}

Carbon stars fall along a well-defined sequence in most infrared color-color diagrams, with each color increasing steadily and monotonically as the DPR increases. The [5.8] $-[8]$ color is an exception, because it is sensitive to both dust content and the strength of molecular absorption in the $5.8 \mu \mathrm{m}$ filter (Srinivasan et al. 2011). Figure 12 plots the [5.8]-[8] color versus $J-K$ in the top panel. From $J-K \sim 1.3$ to 2 , increasing molecular absorption reddens [5.8]-[8], but for 


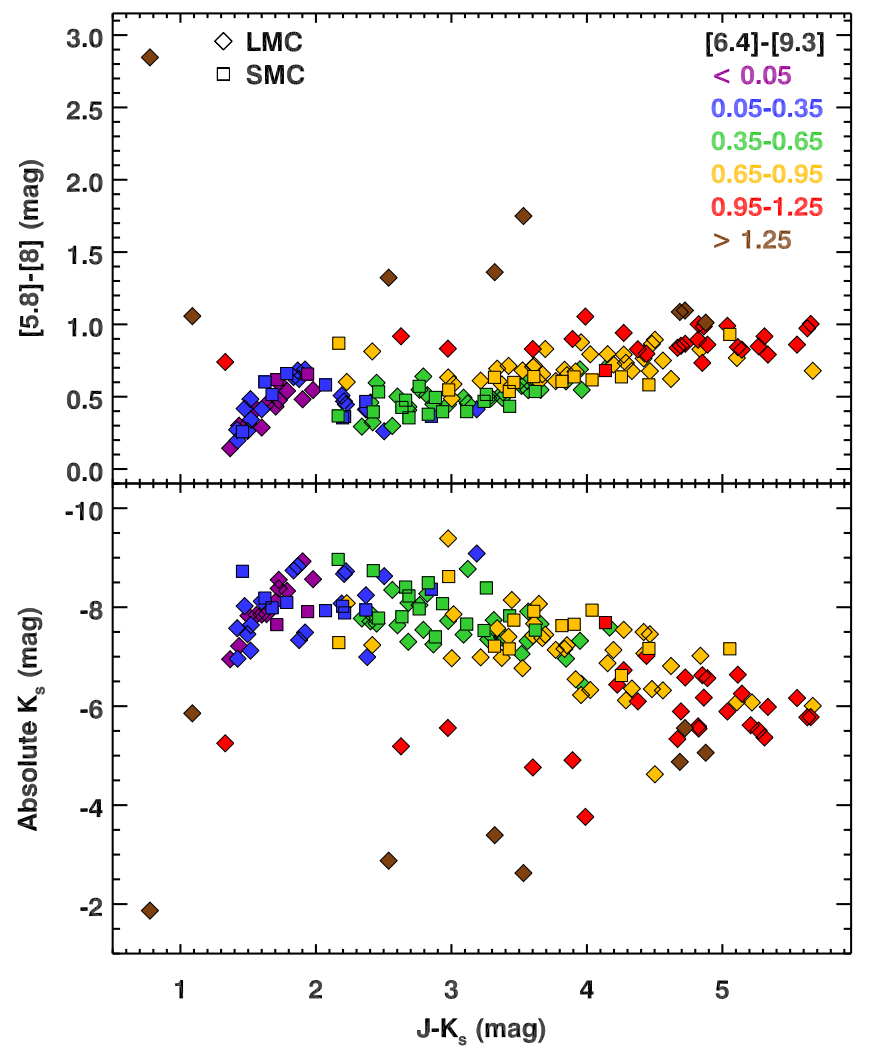

Figure 12. Color-color and color-magnitude diagrams featuring the $J-K_{s}$ colors on the horizontal axis. Symbols are coded for galaxy with shape and for [6.4]-[9.3] color with color. The [6.4]-[9.3] intervals correspond to the CE0-5 classifications. None of the CE5s and only about half of the CE4s follow the sequences. (CE4: $0.95<[6.4]-[9.3]<1.25 ;$ CE5: [6.4] $-[9.3]>1.25$.)

$J-K \gtrsim 2$, increasing dust opacity is the culprit. Sloan et al. (2015b) called these two sequences "molecular" and "dusty" to identify the agent responsible for the reddening. They found that semi-regular variables dominate the molecular sequence $(J-K \lesssim 2$ ), while Mira variables dominate the dusty sequence. This difference is not between overtone and fundamental-mode pulsators, as half of the semi-regulars are pulsating in the fundamental mode. Rather, it is a difference between stars pulsating with small and large amplitudes. Generally, it is the latter that are associated with dust production.

The bottom panel of Figure 12 presents the sample in a more familiar near-IR CMD. Blum et al. (2006) labeled the sequence extending to the red of $J-K_{s} \sim 2$ as the "extreme" AGB stars, ${ }^{18}$ but this terminology is something of a misnomer, as the objects are only extreme in the sense that they are producing carbon-rich dust, something that all carbon stars must do before they end their lives on the AGB. Superlatives like "extreme" are more appropriate for the EROs discovered in the LMC by Gruendl et al. (2008). These are the deeply embedded carbon stars analogous to targets in the Galaxy like AFGL 3068 (e.g., Jones et al. 1977; Lebofsky \& Rieke 1977). We will follow the practice of Sloan et al. (2015b) and refer to objects with $J-K_{s} \gtrsim 2$ as "dusty" carbon stars, by which we mean carbon stars associated with significant and readily measurable quantities of dust.

\footnotetext{
${ }^{18}$ Formally, their criterion was $J-[3.6]>3.1$.
}

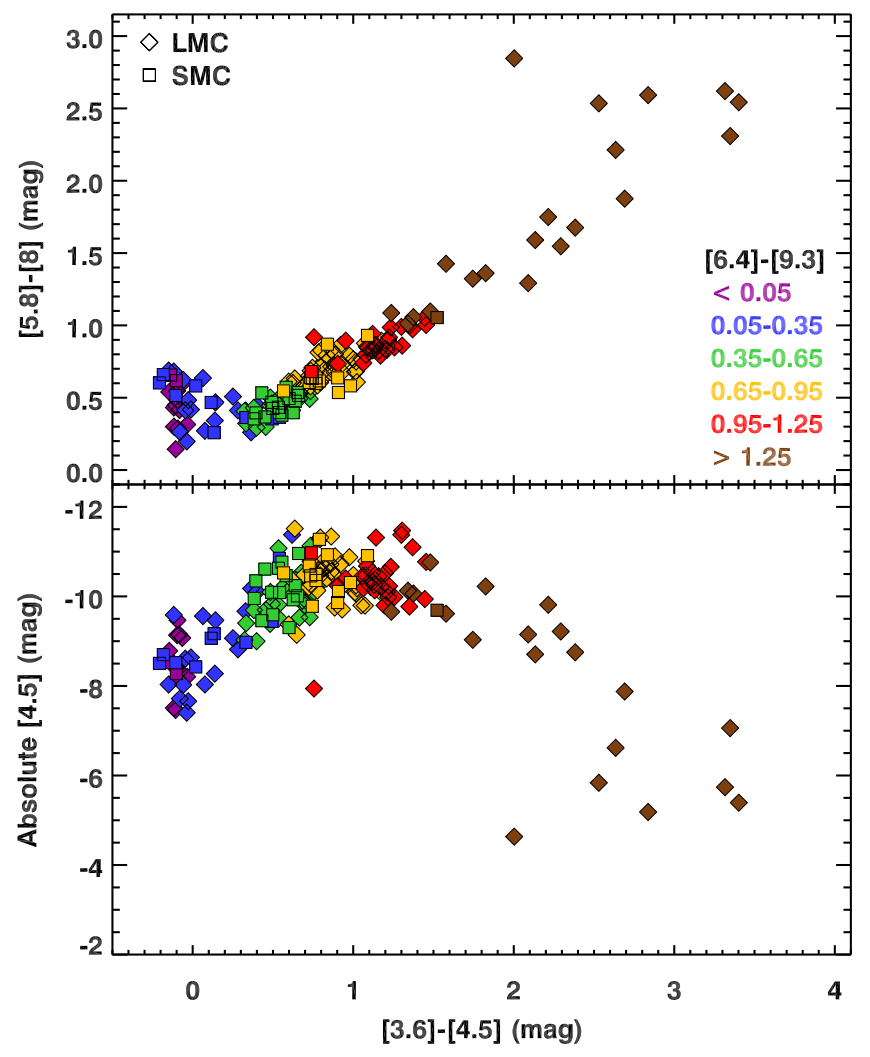

Figure 13. Color-color and color-magnitude diagrams featuring the [3.6][4.5] colors on the horizontal axis. Symbols are coded for galaxy with shape and for [6.4] $-[9.3]$ color with color. The [6.4] $-[9.3]$ intervals correspond to the CE0-5 classifications. Only a handful of the reddest sources in [5.8]-[8] are off the sequence, along with one CE4.

Figure 13 replaces the $J$ and $K_{s}$ filters in Figure 12 with [3.6] and [4.5]. In all four panels of the two plots, the [6.4]-[9.3] color generally increases along the sequence from blue to red, but with some scatter. This scatter appears in any color chosen, and it is consistent with what one would expect from variations in DPR as a function of time, with bluer colors sensitive to warmer and more recently formed dust.

The CE1 sources $(0.05<[6.4]-[9.3]<0.35)$ straddle the boundary between the molecular and dusty sequences in both figures. On the molecular sequence, the overlap between CE0 and CE1 is complete, despite the presence of more dust in the CE1 sources, and it shows that the dust is not determining position on this sequence. The vertical spread of the molecular sequence in the bottom panel of Figure 13 shows that the [3.6] - [4.5] color is less sensitive to molecular bands than $J-K_{s}$.

In Figure 12, none of the CE5 stars $([6.4]-[9.3]>1.25)$ follow the relationship between [5.8]-[8] and $J-K_{S}$ established by the sources with less dust. If they did, they would be off the right-hand edge of the plot, provided we could detect them at $J$ and $K_{s}$. Because these sources are so embedded, if they followed the dusty sequence, they would actually be below the detection limit of the near-IR surveys we examined. Of the 21 CE5 sources, we have valid $J-K_{s}$ colors for only nine, and all appear with bluer $J-K_{s}$ colors than the dusty sequence would lead us to expect. In the bottom panel, all of the CE5 sources plotted are also off the sequence. Their behavior is fully consistent with a shift to bluer $J-K_{s}$ colors. Several of the CE4 and even some CE3 sources can also be seen to have shifted. In Figure 13, no sources are missing, and 


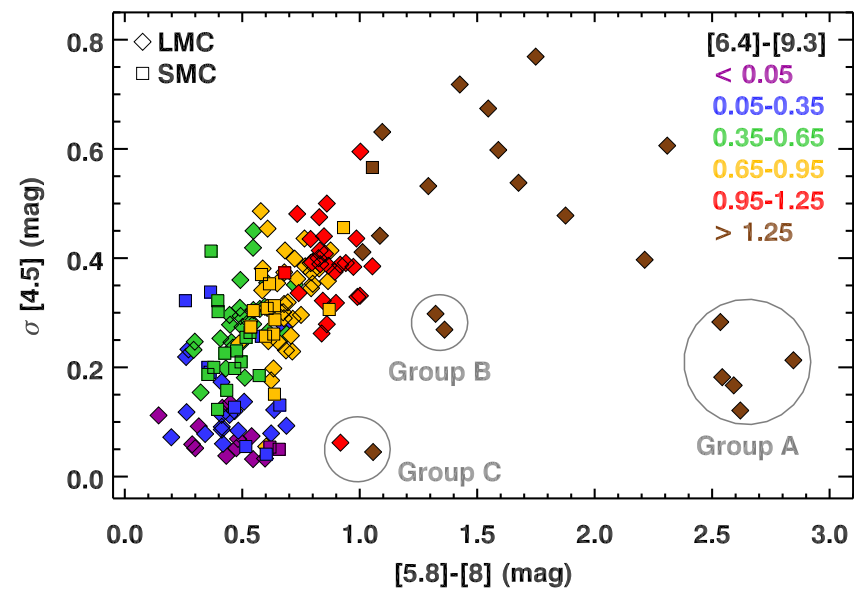

Figure 14. Amplitude of the variability at $4.5 \mu \mathrm{m}$ (as measured by the standard deviation of the IRAC and WISE data) as a function of [5.8]-[8] color. The amplitude increases steadily from the naked carbon stars to the CE4 sources, then drops for those with the reddest [5.8]-[8] and [6.4]-[9.3] colors. The circled groups are discussed in Section 4.6.

the number of off-sequence sources is greatly reduced, with only a few CE5 sources not on the dusty sequence in either panel.

As noted in Section 2.4, it is only for the deeply embedded sources that a photometric mismatch is a danger, and even then the odds of a mismatch are slight for a given source. We conclude that most or all of our off-sequence sources have colors indicative of the source itself and not a chance superposition of two sources in the search beam.

\subsection{Variability}

If the off-sequence sources in Figure 13 have really moved off the AGB, then the amplitude of their variability should have dropped. Figure 14 plots the variability amplitude of all of our carbon stars as a function of [5.8]-[8] color. The values plotted are the standard deviations $(\sigma)$ of the mean magnitudes in Tables 3 and 4. For a pulsation cycle which follows a sine function, the peak-to-peak amplitude $=2 \sqrt{2} \sigma$.

As the stars shift from CE0 (nearly naked) to CE5 (very dusty), the amplitude of their variability increases as they grow redder, but once [5.8]-[8] reaches a value of $\sim 1.5$, the amplitude begins to decline. The three sources furthest from the carbon sequence in the top panel of Figure 13 are all in Group A in the bottom right-hand corner of Figure 14. These are both the reddest and the least variable sources in the sample. More recent photometry of the reddest carbon stars in the LMC at 3.6 and $4.5 \mu \mathrm{m}$ extends the temporal baseline and confirms the trend of decreasing variability with increasing [5.8]-[8] color (B. A. Sargent et al. 2016, in preparation).

Had we chosen to use the variability amplitude at $3.6 \mu \mathrm{m}$ instead of $4.5 \mu \mathrm{m}$, the results would have been the same qualitatively. Dropping the color corrections derived in Appendix A also does not change the result qualitatively. It does change the apparent variabilities quantitatively, increasing the apparent amplitudes for the stars which are most variable because it increases the apparent scatter in the data, but for the relatively non-variable sources, this effect is much smaller. By using the $4.5 \mu \mathrm{m}$ data, we have reduced possible errors from the corrections even more, since the WISE-to-IRAC corrections are much smaller at $4.5 \mu \mathrm{m}$ than at $3.6 \mu \mathrm{m}$.
Four more sources appear to be relatively non-variable for their [5.8]-[8] color, and are labelled as Groups B and C in Figure 14. Group C includes one CE4 source (red diamond), SAGE J054546, which is also off-sequence in the bottom panel of Figure 13. The remaining three are all CE5, and except for unusually low-variability amplitude, none stands out in any other significant way.

\section{DISCUSSION}

\subsection{Evolution off the $A G B$}

The three sources most clearly off the carbon sequence in the color-color diagram in Figure 13 have [5.8]-[8] $>2.5$ and $[3.6]-[4.5]<3$. They are IRAS 05133, IRAS 05191, and IRAS 05260, in order of increasing [3.6]-[4.5] color. The other two sources with [5.8]-[8] $>2.5$ are IRAS 05026 and IRAS 05042. Group A in Figure 14 consists of these five stars. They are much less variable than the rest of the sources with [5.8]-[8] $\gtrsim 1.5$. And all five are also $\mathrm{SiC}$ absorption (CA) sources.

In the top panel of Figure 12, the only CA source with a $J-K_{s}$ color is IRAS 05133, and it is bluer than any other carbon star in our sample! Whatever has led to its unusually blue [3.6]-[4.5] color has allowed us to detect an even bluer source at $J$ and $K_{s}$. With $K_{s}=16.6$ and $J=17.4$, it is just at the detection limit. The other four sources are redder at [3.6] $-[4.5]$, have more extinction in the near-IR, and consequently are undetected at $J$ and $K_{s}$.

Thus the five most embedded carbon stars in our sample (as measured with [5.8]-[8]) have properties consistent with evolution off the AGB. Their variability is lower than the less embedded sources, they show $\mathrm{SiC}$ absorption, indicating high column densities of dust, and three appear to be developing double-peaked SEDs as the central star becomes visible.

However, other stars are relatively non-variable, and others also show $\mathrm{SiC}$ absorption. Of the other non-variable sources, all but SAGE J054546 (CE4) are either CE5 or CA5 (i.e., [6.4] $-[9.3]>1.25)$. These sources may be approaching the end of their AGB evolution.

The steady decline in variability past [5.8]-[8] 1.5 (Figure 14) suggests that more than just the handful of sources most obviously off of the sequences in Figure 13 are approaching the end of their AGB lifetimes.

\subsection{Non-spherical Dust Shells}

For the off-sequence sources, the excess blue flux indicates that we are able to peer into the dust and glimpse the central star, either directly or through scattered light. The dust envelope may be growing patchy or it may be distributed asymmetrically, perhaps in a torus or possibly even a disk.

Figure 12 reveals other sources which do not follow the carbon-star sequence at $J$ and $K_{s}$, even though they are on the sequence in the longer-wavelength filters in Figure 13. In particular, a number of CE4 sources (in red) are off-sequence in both panels of Figure 12, with much bluer $J-K_{s}$ colors than expected for either their [5.8]-[8] colors or $K_{s}$ magnitudes. More of the CE5 sources also behave similarly.

The opacity of amorphous carbon dust drops as $\sim \lambda^{-2}$, making it difficult to explain how we could directly observe the central source at $J$ and $K$ but not at 3.6 and $4.5 \mu \mathrm{m}$. If we are detecting the central source via light scattered above and below a disk or in the polar regions of an asymmetric dust shell, then 
we would expect to see a bluer color at $J-K_{s}$ than at [3.6] -[4.5], because the scattering efficiency drops as $\lambda^{-4}$. BernardSalas et al. (2006) proposed scattering in a system with a disk viewed close to edge-on to explain the unusual object SMP LMC 011, which presents an optically thick carbon-rich dust spectrum with molecular absorption bands in the infrared but shows $\mathrm{H} \alpha$ in emission in the optical. Sloan \& Egan (1995) suggested scattering from the poles of an asymmetric dust distribution in the embedded Galactic carbon star IRC +10216 to explain emission from relatively warm dust above and below the central source. This hypothesis could be tested for the embedded Magellanic carbon stars by measuring their polarization as a function of wavelength in the near-IR.

The nature of the dust geometry has significant consequences. Sloan \& Egan (1995) noted that the deviation from spherical symmetry they argued for in IRC +10216 was subtle and did not prevent spherically symmetric radiative transfer models from working. Disks are a different story. Their nonisotropic emission would lead to significant uncertainties in bolometric luminosities and thus our basic knowledge about the sources. Boyer et al. (2012) noted that the ten dustiest AGB stars detected in the SAGE surveys of the SMC accounted for $17 \%$ of the total dust input to the galaxy when assuming spherical symmetry. If the dust in embedded carbon stars were in disks and not outflowing shells, the amount of dust produced by AGB stars would have to be revised downward, because the DPR scales with outflow velocity, which in disks would be zero.

Disks imply binarity, which leads to more consequences. Equatorial disks can result from binaries interacting during the AGB phase (e.g., Soker \& Livio 1989). If the sources we have identified as off-sequence are interacting binaries, then they may have have never been on sequence and are instead following a different evolutionary path in color-color space. High-resolution imaging of Galactic sources show that binary interactions are not rare at all. The Mira variable $\mathrm{R} \mathrm{Scl}$ is embedded in a spiral structure indicative of interactions of the outflows from the carbon star with a companion (Maercker et al. 2012). $\mathrm{L}^{2}$ Pup, the nearest AGB star in the sky, has a dusty disk, evidence of a companion, and the beginnings of bipolar nebulosity (Kervella et al. 2014, 2015). These observations add to the evidence that binaries and their interactions on the AGB strongly influence the structure of post-AGB objects and planetary nebulae (PNe) (e.g., Lagadec \& Chesneau 2014; Jones 2015).

As already stated, near-IR polarimetry of the most embedded carbon stars in the present sample would help diagnose the geometry of these systems. Radiative transfer modeling would also help by translating the constraints imposed by the SEDs to constraints on the dust morphology.

\subsection{SiC and Luminosity}

Carbon stars in the SMC generally show very little SiC emission compared to their counterparts in the LMC and the Galaxy, but there are exceptions. Sloan et al. (2006) found five stars in the SMC that had significantly more $\mathrm{SiC}$ for their [6.4] -[9.3] color, and the present sample adds two more SiC-rich sources, both with bluer [6.4]-[9.3] colors than the other five (see Figure 5). Sloan et al. (2006) examined a number of properties for the five SiC-rich stars in their sample and could not find anything that distinguished them besides their $\mathrm{SiC}$ emission strength.

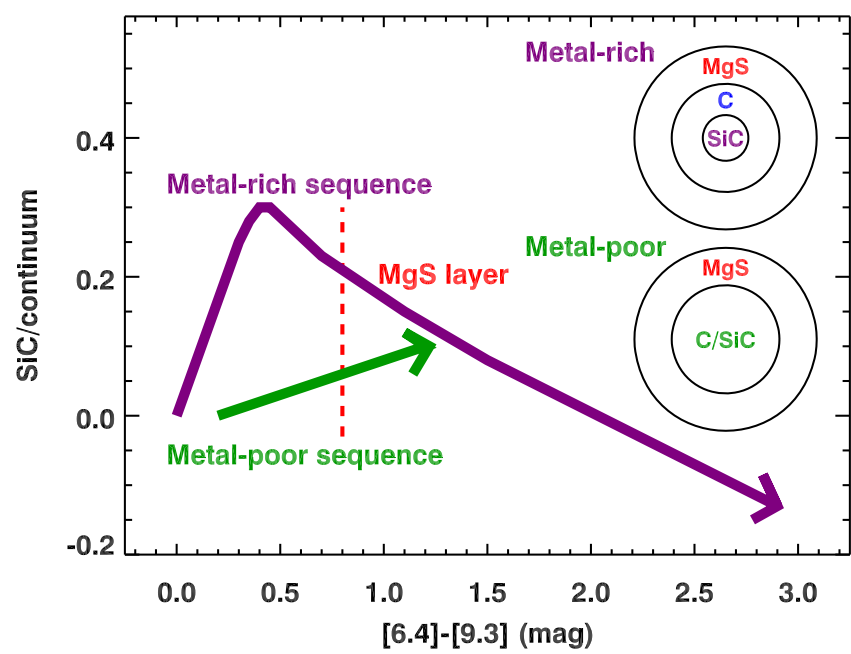

Figure 15. Possible scenario for the evolution of dust grains, modeled on a figure by Leisenring et al. (2008). The dust around more metal-rich stars tends to follow the upper track, with a rapid growth in $\mathrm{SiC}$ emission with increasing [6.4]-[9.3] color, then a steady decline until the feature finally goes into selfabsorption in the most optically thick shells. Around more metal-poor stars, the $\mathrm{SiC}$ emission grows much more gradually as the dust shells redden. $\mathrm{MgS}$ emission begins at different [6.4]-[9.3] colors, depending on the metallicity of the star.

To compare the $\mathrm{SiC}$-rich and $\mathrm{SiC}$-poor sources in the current sample, we concentrate on [6.4]-[9.3] colors between 0.35 and 0.95 (CE2-3), where the two tracks are most distinct, and consider the sources above and below $\mathrm{SiC} /$ continuum $=0.125$ separately. In the SMC, the mean bolometric magnitude for the $\mathrm{SiC}$-rich group is $-5.05 \pm 0.41$, compared to $-5.08 \pm 0.50$ for the $\mathrm{SiC}$-weak group. The uncertainties in the mean are 0.17 and 0.11 , respectively. The two groups are indistinguishable in luminosity, which implies that they have similar mass, age, and metallicity distributions.

In the $\mathrm{LMC}$, the $\mathrm{SiC}$-rich group has $M_{\mathrm{bol}}=-4.91 \pm 0.45$, versus $-5.20 \pm 0.53$ for the $\mathrm{SiC}$-poor group. The uncertainties in the mean are 0.08 and 0.09 , respectively. While the separation is larger and more statistically significant, it is in the opposite sense to what we might expect, with the more luminous stars, which presumably would be more massive and more metal-rich, associated with the weaker SiC features.

Thus, while the relative numbers of stars showing strong and weak SiC emission in the Galaxy, LMC, and SMC support the hypothesis that the strength of the $\mathrm{SiC}$ feature is tracing metallicity, we find no support for the hypothesis within a given galaxy. Sloan et al. (2006) were unable to explain why some sources in the SMC showed strong SiC emission while most did not, and we are unable to improve on that situation.

\subsection{Layered Grains}

Lagadec et al. (2007) suggested that the condensation sequence of $\mathrm{SiC}$ and amorphous carbon differed in metal-rich and metal-poor stars, with $\mathrm{SiC}$ forming first in the Galaxy and later in the SMC. Leisenring et al. (2008) followed with a proposal for multiple evolutionary tracks for dust grains, with the extremes determined by whether $\mathrm{SiC}$ or amorphous carbon formed first. Figure 15 simplifies the proposal of Leisenring et al. (2008) and presents two tracks consistent with the infrared properties of Galactic and Magellanic carbon stars. The key is that the dust grains must be layered, which may 
wreak havoc with attempts to use the grain properties to estimate the masses of the refractory elements in dust grains.

$\mathrm{MgS}$ is a case in point. $\mathrm{MgS}$ grains do not form until the dust shells pass an optical thickness limit which depends on the metallicity of the sample, as Figures 6 and 7 show. The MgS could condense as grains independently, but then, in order to explain the strength of the MgS feature in post-AGB objects and young $\mathrm{PNe}$, more $\mathrm{S}$ would be required than can exist around these stars. On these grounds, Zhang et al. (2009) argued that $\mathrm{MgS}$ could not be the carrier of the $26-30 \mu \mathrm{m}$ feature.

Zijlstra et al. (2006) proposed that $\mathrm{MgS}$ formed as a layer on pre-existing seeds of $\mathrm{SiC}$ and amorphous carbon. Lombaert et al. (2012) argued that the optical properties of a grain coated with $\mathrm{MgS}$ would mimic those of a solid $\mathrm{MgS}$ grain, which would account for the strength of the $\mathrm{MgS}$ feature in PNe without violating abundance constraints. Sloan et al. (2014) explained why other concerns raised about $\mathrm{MgS}$ as the carrier of the 26-30 $\mu \mathrm{m}$ feature can be discarded. All of the evidence is consistent with $\mathrm{MgS}$ forming a layer on carbonaceous grains, with the condensation trigger depending on metallicity.

However, it is unlikely that any of the layers are pure. The metal-rich track suggests that metal-rich carbonaceous grains should consist of $\mathrm{SiC}$ cores surrounded by amorphous carbon mantles. In the most optically thick dust shells, these would be mostly coated by $\mathrm{MgS}$, and yet the spectra from these sources show absorption from $\mathrm{SiC}$. Thus some $\mathrm{SiC}$ must be present within one optical depth of the surface of the grains, which means that not all grains are coated with $\mathrm{MgS}$, or the coatings are incomplete. Furthermore, if the grains consist of a $\mathrm{SiC}$ core and an amorphous carbon mantle, that mantle cannot be pure and must also contain some SiC.

Young carbon-rich PNe in the LMC and SMC often show unusually strong emission features at $\sim 11.5 \mu \mathrm{m}$ in their spectra (Bernard-Salas et al. 2009). Sloan et al. (2014) showed that the shape of these features was consistent with SiC dust, modified by the presence of emission from polycyclic aromatic hydrocarbons. From abundance arguments, one would expect weaker $\mathrm{SiC}$ emission in more metal-poor objects, but the opposite is true, with carbon-rich Magellanic PNe showing stronger SiC emission than in the Galaxy. Sloan et al. (2014) suggested that photoprocessing of carbonaceous grains would preferrentially remove amorphous carbon from the surface, resulting in grains coated by $\mathrm{SiC}$. Such a scenario would require some intermixing of $\mathrm{SiC}$ and amorphous carbon in the outer layers of the grains.

The available observational evidence points to layered grains with a structure more complicated than depicted in Figure 15. The strength of the $\mathrm{SiC}$ and $\mathrm{MgS}$ features with increasing [6.4] - [9.3] color points to two tracks as shown, but the presence of $\mathrm{SiC}$ absorption in the most embedded sources and the strength of the $\mathrm{SiC}$ feature in young $\mathrm{PNe}$ requires some $\mathrm{SiC}$ in the layers of the grains within one optical depth of the surface.

\subsection{Completeness}

The pointed nature of spectroscopic samples introduces biases, which can make it challenging to compare different populations. This work has focused on using properties like the pulsation period of the star or the [6.4]-[9.3] color, which is a proxy for the amount of dust, as the independent variable in order to compare sources with similar properties when examining other properties such as the strength of emission

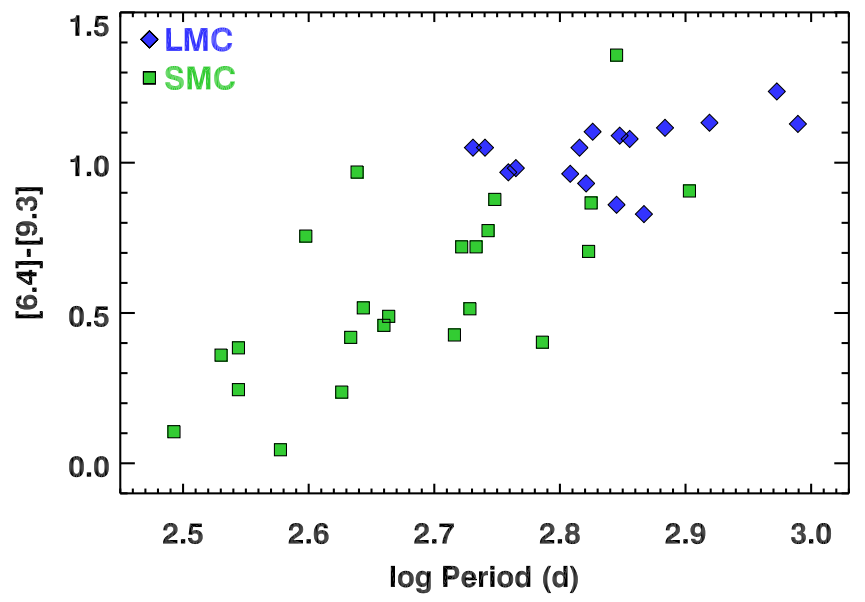

Figure 16. Samples of Magellanic carbon stars studied by van Loon et al. (2006, 2008) plotted on the [6.4]-[9.3] vs. period plane. The SMC sample shows less dust than the LMC sample because it generally probes a sample with lower pulsation periods. Both samples follow the same trend of increasing dust content with increasing pulsation period.

features from $\mathrm{SiC}$ or $\mathrm{MgS}$ dust. A key result is that the amount of dust produced by a star in the Galaxy, LMC, or SMC, depends primarily on its pulsation period and initial mass. The effect of metallicity is unclear with the current sample. While we do see hints of less dust in more metal-poor stars when controlling for period and mass, this decrease is not statistically significant in the IRS samples.

In contrast, van Loon et al. (2008) found a clearer difference in the dust content of their sample of carbon stars in the SMC, compared to a similar study in the LMC (van Loon et al. 2006). They drew this conclusion from a spectroscopic study of the absorption bands from $\mathrm{C}_{2} \mathrm{H}_{2}$ at 3.1 and $3.8 \mu \mathrm{m}$, finding that dust veiling affected the bands more in the LMC than in the SMC. Figure 16 plots the [6.4]-[9.3] color for the stars in common between their sample and ours as a function of pulsation period and shows that while the carbon stars in the LMC have more dust on average than their counterparts in the SMC, the two samples have different distributions in pulsation period, as well. None of the carbon stars in the LMC in common with the IRS sample have a period less than 500 days, compared to over half the SMC sample. Given the differences in the LMC and SMC samples, one would expect the stars in the LMC to show more dust, simply because they are pulsating with longer periods.

The IRS-based studies have concentrated on comparing like with like, which means comparing the dust content in stars with similar pulsation periods. Previous studies with the IRS on Spitzer have found little dependence of the dust content on metallicity in carbon stars. The larger sample considered here has not changed that result.

However, the evidence is building that the populations of carbon stars in LMC and SMC differ. Figure 16 shows that the $3 \mu \mathrm{m}$ samples contain more longer-period pulsators and dustier carbon stars in the LMC than the SMC. The IRS samples show a similar difference in the two galaxies. In the SMC sample, the reddest [6.4]-[9.3] color is 1.4, so that only one SMC source out of 40, NGC 419 MIR 1, is classified as CE5, compared to 20 CE5 and CA5 sources out of 144 in the LMC, 15 of which are redder than NGC 419 MIR 1, with [6.4]-[9.3] colors extending past 3.0. At first glance, this could easily be a selection effect, as is often the case for spectroscopic samples, 
since the carbon-rich component of at least three IRS programs focused exclusively on the most embedded objects in the LMC. However, analogous objects do not appear to be present in the SMC. Our searches for deeply embedded carbon stars in the SMC using photometry from Spitzer and WISE have been unsuccessful. Searches using mid-IR photometry from Spitzer confirm this result (Dell'Agli et al. 2015; Srinivasan et al. 2016), and searches in the far-IR with Herschel have also not uncovered any deeply embedded AGB stars in the SMC (Jones et al. 2015). These missing objects are likely to be massive carbon stars not present in the SMC due to a lower rate of recent star formation (e.g., Ventura et al. 2016).

This deficiency of embedded stars in the SMC combined with how these sources move off the carbon sequence in nearIR color-magnitude space solves a problem first noticed by Lagadec et al. (2010). They derived a relation between absolute magnitude at $K_{s}$ and $J-K_{s}$ as a means of estimating the distances to carbon stars, but they found much more scatter in the LMC sample than the SMC and used the latter galaxy to derive a relation. Figure 12 shows that the off-sequence embedded carbon stars that appear only in the LMC sample are responsible for this apparent scatter. This new finding presents a new problem for the use of color-magnitude relations to find the distance to carbon stars, because a given star might be off sequence. A possible solution would be to develop and use color-magnitude relationships at multiple wavelengths to estimate distance and to remove outliers at shorter wavelengths. Alternatively, off-sequence stars could be identified from their reduced variability.

\subsection{The Dust-production Trigger}

The dichotomy between the carbon stars on the molecular and dust sequences described by Sloan et al. (2015b) points to pulsation as the trigger for dust production. The carbon stars producing dust are almost entirely Mira variables, while the carbon stars on the molecular sequence are either pulsating in one or more overtones, or they are pulsating weakly in the fundamental mode. The link between strong pulsation and carbon-rich dust production is clear, even if which is the cause and which is the effect is not.

Abundance arguments suggest that dredge-up is the trigger. As described in the introduction, similar quantities of dredgedup carbon will lead to a higher amount of free carbon in a more metal-poor star (Matsuura et al. 2005; Sloan et al. 2012). The DPR should be related to the free carbon abundance, and yet we clearly do not observe higher DPRs in more metal-poor carbon stars. Sloan et al. (2012) suggested that once the free carbon abundance crossed a critical threshold, dust production would spike, stripping the stellar envelope and ending its evolution on the AGB. Lagadec \& Zijlstra (2008) argued similarly, suggesting that the final dredge-up on the AGB would initiate a superwind. Crossing a free-carbon threshold is the more likely trigger.

Dredge-ups affect atmospheric opacity and generate more consequences than just free-carbon abundance. Higher opacities will increase the stellar radius, lowering the escape velocity and making dust-production and mass-loss easier. An expanded and more opaque atmosphere may also be more unstable to pulsations, which would in turn push material out at velocities closer to the now lowered escape velocity. In other words, the higher pulsation amplitudes and increased dust production may both result from the same underlying cause, the last critical dredge-up on the AGB.

What is intriguing about the sample of IRS-observed Magellanic carbon stars is that it may include some sources on the other side of that final superwind. Because of the selection biases that defined this sample, it cannot provide meaningful relative statistics of carbon stars in different phases of their lifetimes on the AGB. But it can inform the photometric studies needed to study these fundamentally important phases of stellar evolution.

\section{SUMMARY}

The IRS on the Spitzer Space Telescope obtained spectra of 144 carbon-rich AGB stars in the LMC and 40 in the SMC. Comparison to Galactic samples reveals no strong dependence of the amount of dust produced with metallicity. Using the bolometric magnitudes to estimate initial mass and accounting for the different mass distributions hints at a subtle decrease in DPR from the LMC to the SMC, but the result is not statistically significant $(1.3 \sigma)$.

The amount of $\mathrm{SiC}$ dust accompanying the amorphous carbon does depend on metallicity, with strong $\mathrm{SiC}$ features observed in most of the Galactic sample and roughly half of the LMC sample. Most of the carbon stars in the SMC show much weaker SiC emission. Some of the SMC stars are exceptions and show stronger $\mathrm{SiC}$ emission, but the reason is unknown. Generally, as the optical depth of the dust increases, the SiC emission rises to a peak and then shifts into self-absorption. The LMC is unique among the three samples considered here in that several sources show the $\mathrm{SiC}$ feature strongly in absorption.

The strength of the $\mathrm{MgS}$ feature at $\sim 30 \mu \mathrm{m}$ also depends on metallicity, with lower metallicity leading to the appearance of the feature in more optically thick dust shells. This behavior is consistent with previous arguments that the $\mathrm{MgS}$ condenses as a layer onto pre-existing grains.

Photometry in the near-IR and at 3.6 and $4.5 \mu \mathrm{m}$ reveals some radiation from the central stars in many of the more embedded carbon stars. This excess blue emission shifts these sources off the usual carbon sequence seen in most infrared color-color diagrams and CMDs. The photometric behavior of these off-sequence stars is consistent with either a patchy dust shell or scattered light in a system with less dust above its poles, possibly a disk.

The pulsation amplitudes generally increase as the dust grows optically thick. However, the most embedded sources in our sample are relatively non-variable, show $\mathrm{SiC}$ absorption in their spectra, and also show signs that they are revealing their central star. This behavior is consistent with evolution off the AGB. Their observed properties are also consistent with interacting binary systems, which complicates their interpretation.

We thank the anonymous referee for helpful and constructive comments. GCS was supported by NASA through Contract Number 1257184 issued by the Jet Propulsion Laboratory, California Institute of Technology under NASA contract 1407 and the NSF through Award 1108645. FK received support from the Ministry of Science and Technology (MoST) of Taiwan, grant MOST104-2628-M-001-004-MY3. This research relied on the following resources: NASA's Astrophysics Data System, the Infrared Science Archive at the 


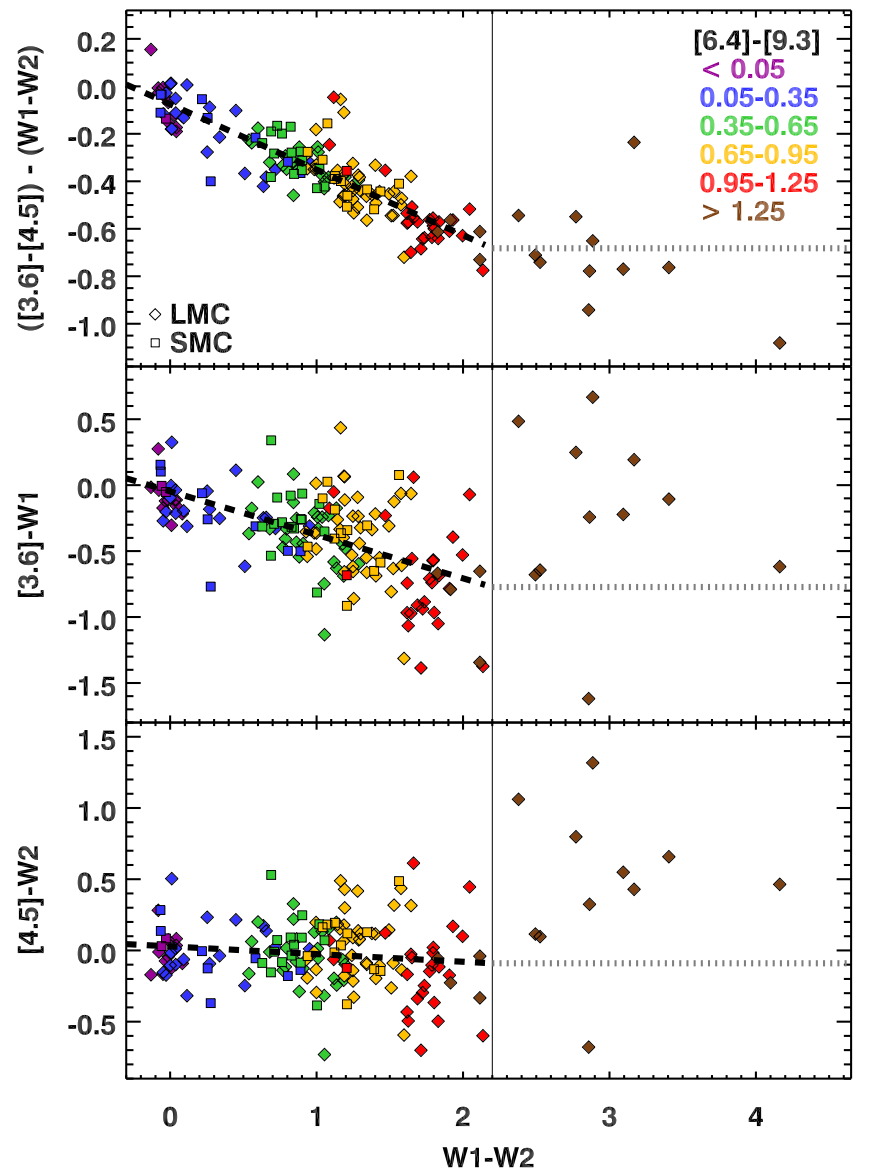

Figure 17. Relations between IRAC and WISE colors and magnitudes, as a function of W1-W2 color, for carbon stars. Lines are fitted to the data with W1$W 2<2.2$.

Infrared Processing and Analysis Center, operated by JPL, and the Simbad and VizieR databases, operated at the Centre de Données astronomiques de Strasbourg.

\section{APPENDIX A}

\section{PHOTOMETRIC RELATIONS FOR CARBON STARS}

The repeated infrared photometric surveys of the Magellanic Clouds provide multiple epochs in the IRAC and WISE filter systems. Because the WISE filters at 3.4 and $4.6 \mu$ m overlap the IRAC filters at 3.6 and $4.5 \mu \mathrm{m}$, it is possible to develop colorbased transformations so that we can convert the WISE data to the IRAC system. The result is up to ten or more epochs over baselines of several years for some of the targets in our sample.

Figure 17 plots the differences between the IRAC and WISE systems as a function of the $W 1-W 2$ color ([3.4]-[4.6]). The top panel shows that for carbon stars, the difference between IRAC [3.4]-[4.5] and WISE colors is well behaved. We could fit the entire sequence with a quadratic, but instead fit a line for $W 1-W 2<2.2$.

The lower two panels in Figure 17 illustrate the need for a cut-off at $W 1-W 2=2.2$. To the blue, the relations of [3.6] to $W 1$ and [4.5] to $W 2$ are clear enough, although with significant noise due to our limited sampling and the variability of the sources. This variability is suppressed in the top panel because the color changes much less than the magnitudes over the pulsation cycle. In the lower two panels, however, the data to the red of $W 1-W 2=2.2$ do not conform to the behavior of the
Table 8

Color and Magnitude Corrections for Carbon Stars

\begin{tabular}{lrc}
\hline \hline Relation & $y$-intercept & Slope \\
\hline$([3.6]-[4.5])-(W 1-W 2)$ versus $W 1-W 2$ & -0.0752 & -0.2759 \\
{$[3.6]-W 1$ versus $W 1-W 2$} & -0.0469 & -0.3298 \\
{$[4.5]-W 2$ versus $W 1-W 2$} & 0.0283 & -0.0539 \\
\hline
\end{tabular}

Note. These corrections are only valid for $W 1-W 2<2.2$.

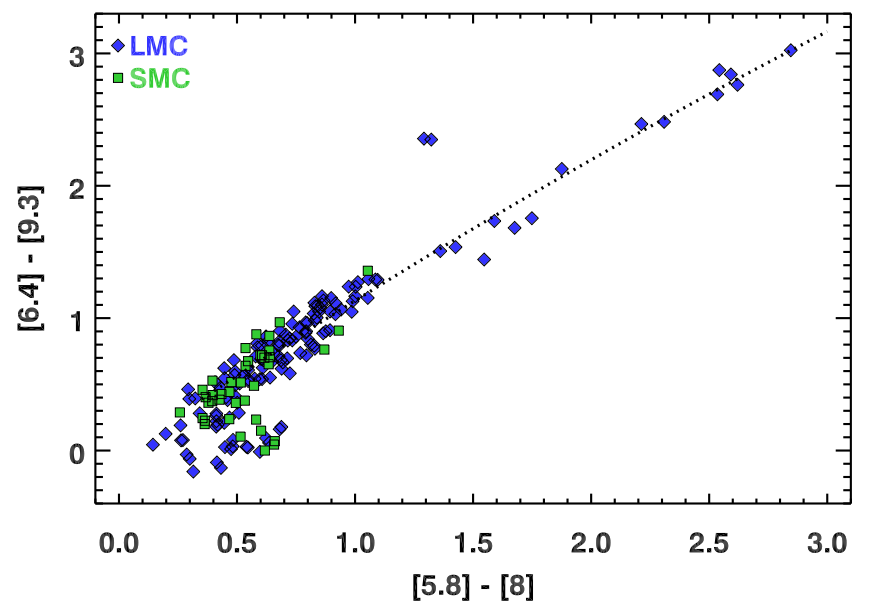

Figure 18. Relationship between [6.4]-[9.3] and [5.8]-[8] colors and a quadratic fitted to data with $[6.4]-[9.3]>0.35$. The two data points at [6.4] $-[9.3] \sim 2.3$ and [5.8]-[8] 1.3 are badly mispointed, resulting in artificially red [6.4]-[9.3] colors, which have been replaced in the data tables using estimates from the fitted relation (see text).

bluer sources, with all but one data point falling above any line fitted to the blue data. An examination of Figure 14 hints at what must be happening. All of the stars with $W 1-W 2>2.2$ are classified as CE5 or CA5. From CE0 to CE4, the pulsation amplitude increases steadily, but most of the CE5s are on the other side of the peak amplitude. These stars are likely to be near the end of their AGB lifetimes and may be exhibiting nonspherical dust geometries. For lack of a better solution for the redder sources, we will assume that the corrections are flat past $W 1-W 2=2.2$ when combining WISE and IRAC data to determine mean magnitudes at 3.6 and $4.5 \mu \mathrm{m}$.

Because some sources are missing data in the different filters involved, the corrections determined for individual magnitudes do not quite produce the correction for color when combined algebraically. We have modified the magnitude corrections slightly so that they do. Table 8 gives the color-based corrections for all three panels in Figure 17. The shift between [4.5] and $W 2$ is flatter than between [3.6] and $W 1$ because the longer-wavelength filters overlap more.

Figure 18 illustrates the relationship between the IRAC [5.8] - [8] color and the [6.4]-[9.3] color derived from the IRS spectra. We excluded the two outliers with [5.8]-[8] 1.3 when fitting a quadratic to the data. The polynomial coefficients are $-0.0282,1.2074,-0.0476 .{ }^{19}$ The standard deviation of the data about this function is 0.1085 .

The two outliers are IRAS 04589 and IRAS 05306, both from Program 37088. The IRS observations were centered on a nearby 2MASS star and were somewhat mispointed for the intended target, leading to a loss of $75 \%-80 \%$ of the flux in SL

\footnotetext{
${ }^{19}$ Polynomical coefficients are listed with the $y$-intercept first.
} 


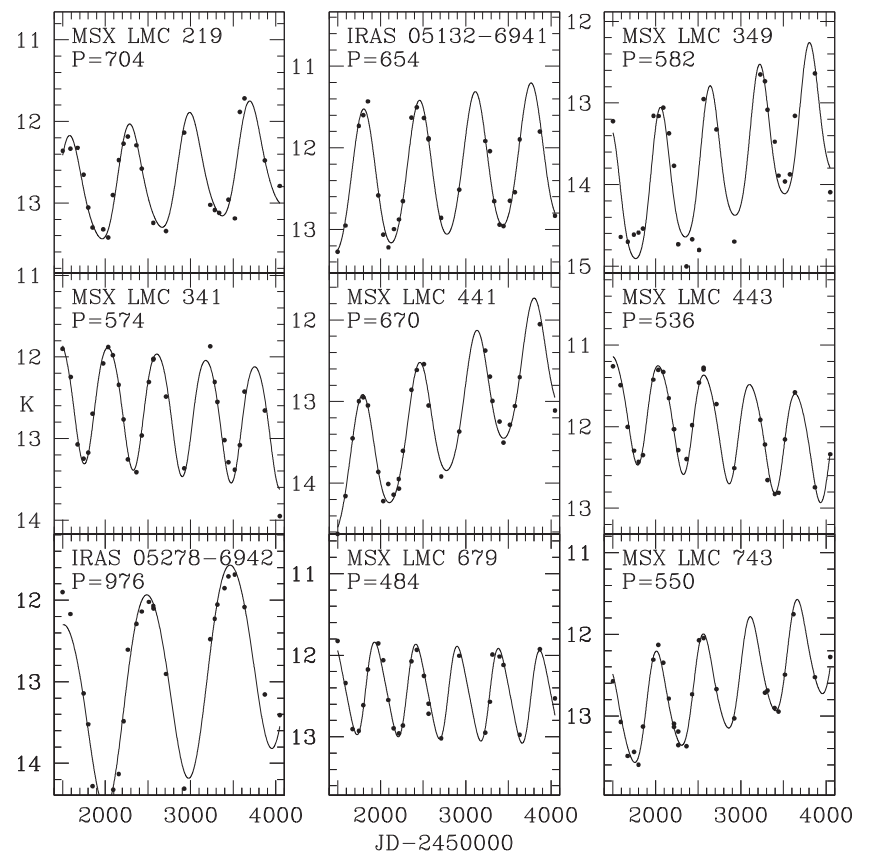

Figure 19. Light curves at $K$ for the nine objects with newly reported pulsation periods. The solid points are the measured $K$ values and the lines are fits to the light curves using the periods displayed in the individual panels. The range on the $K$ axis is set to 3.2 mag for all objects so that the differences in amplitudes are readily apparent.

compared to LL. As a result, the targets did not appear as point sources in the spectral images, the optimal extraction broke down, and we used the tapered-column extraction method for these sources. The truncation of starlight by the spectroscopic slit in such a case is a function of wavelength, leading to an artifically reddened spectrum and a false measurement of the [6.4]-[9.3] color. For this reason, we replaced the [6.4]-[9.3] color for these two sources in all data tables and figures with values estimated from their [5.8]-[8] color. ${ }^{20}$

\section{APPENDIX B NEW PULSATION PERIODS FROM $K$-BAND PHOTOMETRY}

Some of the targets for Spitzer program 3505 (PI P. Wood) were very red and had no light curves available in the MACHO or OGLE monitoring programs. ${ }^{21} \mathrm{P}$. Wood led a long-term program to monitor many embedded evolved stars in the Magellanic Clouds using CASPIR, the Cryogenic Array Spectrometer Imager (McGregor et al. 1994), in the near-IR on the $2.3 \mathrm{~m}$ telescope of the Australian National University at Siding Spring Observatory. Groenewegen et al. (2007) describe the observational and data reduction procedures, and Kamath et al. (2010) give the $K$ - and $L$-band light curves of NGC 419 IR 1, NGC 419 MIR 1, NGC 1978 IR 1, and NGC 1978 MIR 1. Figure 19 presents the light curves at $K$ for nine additional objects. The fitted light curves in the figure were

\footnotetext{
${ }^{20}$ IRAS 04589 and IRAS 05306 have [5.8] - [8] colors of 1.292 and 1.323, respectively, giving estimated [6.4]-[9.3] colors of 1.452 and 1.486 , compared to measurements of 2.356 and 2.349 from the mispointed spectra.

21 The MACHO project searched for gravitational lensing events from massive compact halo objects in the direction of the Magellanic Clouds. Fraser et al. (2005) show how this monitoring facilitated the study of long-period variables. The OGLE project has operated similarly.
}

Table 9

Multi-epoch $K$-band Photometry

\begin{tabular}{lcc}
\hline \hline Target & JD-2450000 & $K(\mathrm{mag})$ \\
\hline MSX LMC 219 & 1501 & 12.36 \\
& 1591 & 12.34 \\
& 1675 & 12.32 \\
& 1746 & 12.65 \\
& 1800 & 13.05 \\
& 1852 & 13.30 \\
& 1976 & 13.32 \\
& 2035 & 13.42 \\
& 2090 & 12.90 \\
2158 & 12.47 \\
\hline
\end{tabular}

(This table is available in its entirety in machine-readable form.)

made using a Fourier series of a single frequency and its first harmonic together with a term allowing a linear variation of $K$ with time.

These objects clearly show large pulsation amplitudes of 1.1-2.5 mag in $K$ as well as long-term variations in $K$ of up to $1 \mathrm{mag}$. The latter variations are short segments of long-term variations (e.g., Whitelock et al. 2003) that are probably due to the episodic nature of dust-ejection mechanisms (e.g., Winters et al. 1994). The large variations evident in the $K$ light curves highlight the fact that variability will introduce a significant scatter in quantities such as $M_{\text {bol }}$ when it is computed from SEDs constructed from single-epoch photometry taken in different bands at different times. Similarly, colors computed using magnitudes obtained at different times for the two bands involved will also show a significant scatter due to variability.

Table 1 identifies the nine new periods determined from $K$ band monitoring with the reference "Appendix B." Table 9 gives the multi-epoch $K$-band photometry.

\section{APPENDIX C NEW PULSATION PERIODS FROM 3 TO $5 \mu \mathrm{m}$ PHOTOMETRY}

All the objects in this study have been observed multiple times at 3.6 and $4.5 \mu \mathrm{m}$ by Spitzer and at 3.4 and $4.6 \mu \mathrm{m}$ by WISE (see Section 2.4). We examined if sufficient epochs were available from the two spacecraft to generate mid-IR light curves and from these determine pulsation periods.

In order to maximize the number of points in each light curve, we converted the data at 3.4 and $4.6 \mu \mathrm{m}$ from WISE to the Spitzer wavelengths using the transformations from Appendix A. Table 10 presents the multi-epoch photometry at 3.6 and $4.5 \mu \mathrm{m}$, including the converted WISE photometry, for all of the Magellanic carbon stars in our sample.

A Fourier fit consisting of a single frequency was made to the resulting light curves at 3.6 and $4.5 \mu \mathrm{m}$. We found that reliable results were only possible for light curves with at least eight individual epochs and a range in the photometry exceeding $\sim 0.5 \mathrm{mag}$. In addition, the periods determined independently at 3.6 and $4.5 \mu \mathrm{m}$ had to agree to better than $5 \%$.

With the above constraints, we were able to fit the light curves of 42 objects. Of these objects, 37 had periods previously determined from more extensive light curves available at shorter wavelengths, such as those from OGLE or those discussed in Appendix B. Comparing the average of the periods from 3.6 and $4.5 \mu \mathrm{m}$ with the previously known 
Table 10

Multi-epoch 3-5 $\mu \mathrm{m}$ Photometry

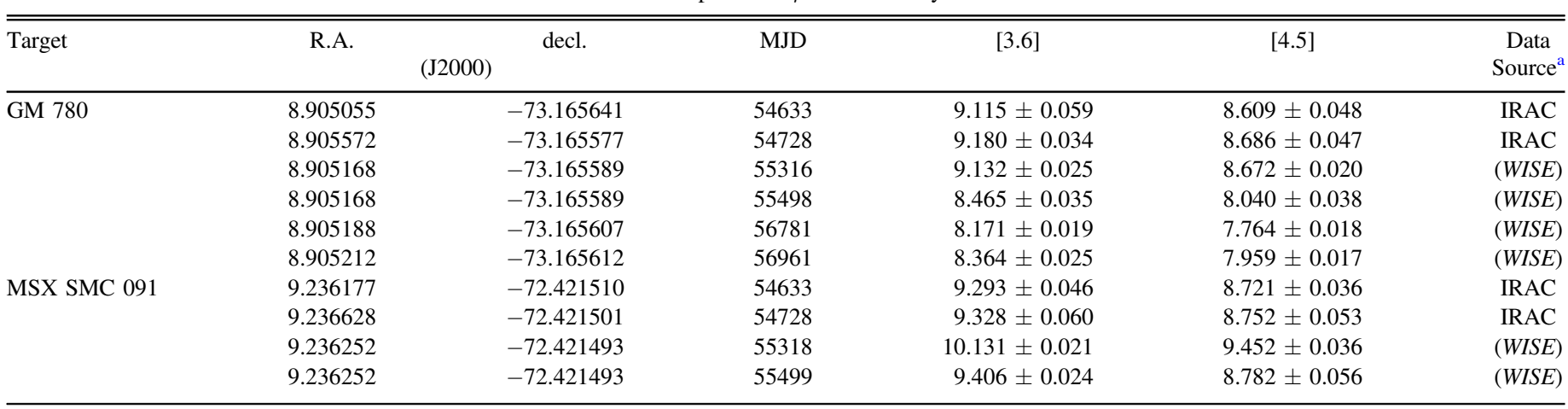

Note.

${ }^{\mathrm{a}}$ WISE data are corrected as described in Appendix A.

(This table is available in its entirety in machine-readable form.)
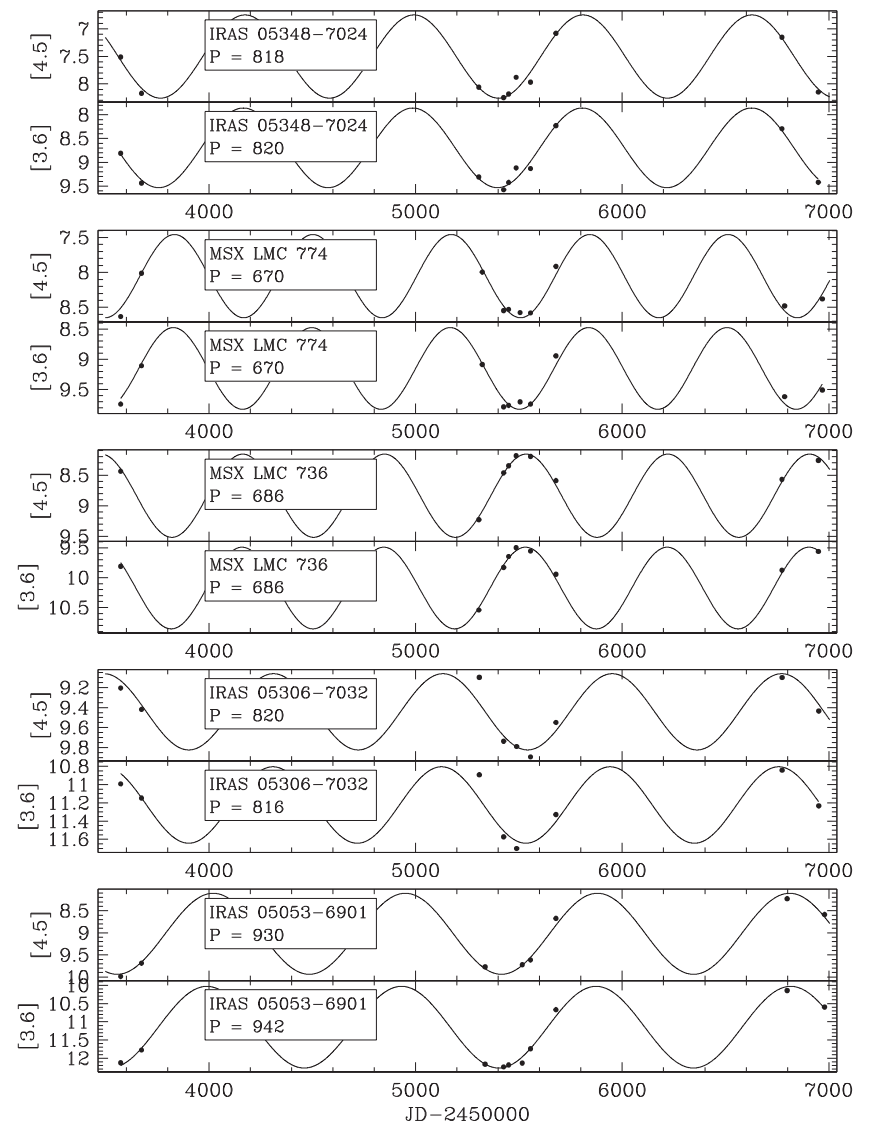

Figure 20. Light curves at 3.6 and $4.5 \mu \mathrm{m}$ of five objects with previously unknown periods. The solid points are the measured values and the lines are sine functions fitted to the light curves using the periods displayed in the panels.

periods showed that 31 of the 37 periods agreed to better than $5 \%$, a success rate of $84 \%$.

In our sample of fitted periods we found five sources with previously unknown periods. Figure 20 plots their light curves. The averages of the periods from 3.6 and $4.5 \mu \mathrm{m}$ appear in Table 1, with the reference for the periods given as "Appendix C." Based on our comparison of periods in the literature for the 37 other sources, we would expect that at least four of these five new periods are reliable to better than $5 \%$.

\section{REFERENCES}

Bernard-Salas, J., Peeters, E., Sloan, G. C., et al. 2006, ApJL, 652, L29 Bernard-Salas, J., Peeters, E., Sloan, G. C., et al. 2009, ApJ, 699, 1541 Blum, R. D., Mould, J. R., Olsen, K. A., et al. 2006, AJ, 132, 2034 Bolatto, A. D., Simon, J. D., Stanimirović, S., et al. 2007, ApJ, 655, 212 Boyer, M. L., Srinivasan, S., Riebel, D., et al. 2012, ApJ, 748, 40 Buchanan, C. L., Kastner, J. H., Forrest, W. J., et al. 2006, AJ, 132, 1890 Cioni, M.-R., Loup, C., Habing, H. J., et al. 2000, A\&AS, 144, 235 Cutri, R. M., Skrutskie, M. F., van Dyk, S., et al. 2012, yCat, 2281, 0 de Graauw, T., Haser, L. N., Beintema, D. A., et al. 1996, A\&A, 315, L49 Dell'Agli, F., Garcá-Hernández, D. A., Ventura, P., et al. 2015, MNRAS, 454, 4235

Feast, M. W. 2013, in Planets, Stars and Stellar Systems, Vol. 5, ed. T. D. Oswalt, \& G. Gilmore (Dordrecht: Springer), 829

Fraser, O. J., Hawley, S. L., Cook, K. H., \& Keller, S. C. 2005, AJ, 129, 768 Gardiner, L. T., \& Hawkins, M. R. S. 1991, MNRAS, 251, 174

Girardi, L., Rubele, S., \& Kerber, L. 2009, MNRAS, 394, L74

Glatt, K., Grebel, E. K., Sabbi, E., et al. 2008, AJ, 136, 1703

Goebel, J. H., \& Moseley, S. H. 1985, ApJL, 290, L35

Gordon, K. D., Meixner, M., Meade, M. R., et al. 2011, AJ, 142, 102

Groenewegen, M. A. T., Sloan, G. C., Soszyński, I., \& Petersen, E. A. 2009, A\&A, 506, 1277

Groenewegen, M. A. T., Wood, P. R., Sloan, G. C., et al. 2007, MNRAS, 376,313

Gruendl, R. A., Chu, Y.-H., Seale, J. P., et al. 2008, ApJL, 688, L9

Gullieuszik, M., Groenewegen, M. A. T., Cioni, M.-R. L., et al. 2012, A\&A, 537,105

Houck, J. R., Roellig, T. L., van Cleve, J., et al. 2004, ApJS, 154, 18

Jones, B., Merrill, K. M., Puetter, R. C., \& Willner, S. P. 1977, AJ, 83, 1437 Jones, D. 2015, in EAS Publications Ser. 71-72, The Physics of Evolved Stars: A Conf. Dedicated to the Memory of Olivier Chesneau, ed. E. Lagadec, F. Millour, \& T. Lanz (Les Ulis: EDP Sciences), 113

Jones, O. C., Meixner, M., Sargent, B. A., et al. 2015, ApJ, 811, 145

Kamath, D., Wood, P. R., Soszyński, I., \& Lebzelter, T. 2010, MNRAS, 408, 522

Kato, D., Nagashima, Ch., Nagayama, T., et al. 2007, PASJ, 59, 615

Kemper, F., Woods, P. M., Antoniou, V., et al. 2010, PASP, 122, 683

Kervella, P., Montargès, M., Lagadec, E., et al. 2015, A\&A, 578, 77

Kervella, P., Montargès, M., Ridgway, S. T., et al. 2014, A\&A, 564, 88

Kessler, M. F., Steinz, J. A., Anderegg, M. E., et al. 1996, A\&A, 315, L27

Kraemer, K. E., Sloan, G. C., Bernard-Salas, J., et al. 2006, ApJL, 652, L25

Kraemer, K. E., Sloan, G. C., Price, S. D., \& Walker, H. J. 2002, ApJS, 140,389

Kraemer, K. E., Sloan, G. C., Wood, P. R., et al. 2005, ApJL, 631, L147

Lagadec, E., \& Chesneau, O. 2014, in ASP Conf. Ser. 497, Why Galaxies Care about AGB Stars III: A Closer Look in Space and Time, ed. F. Kerschbaum, R. F. Wing, \& J. Hron (San Francisco: ASP), 145 Lagadec, E., \& Zijlstra, A. A. 2008, MNRAS, 390, L59

Lagadec, E., Zijlstra, A. A., Mauron, N., et al. 2010, MNRAS, 403, 1331 Lagadec, E., Zijlstra, A. A., Sloan, G. C., et al. 2007, MNRAS, 376, 1270 Lebofsky, M. J., \& Rieke, G. H. 1977, AJ, 82, 646 
Lebouteiller, V., Bernard-Salas, J., Sloan, G. C., \& Barry, D. J. 2010, PASP, 122,231

Leisenring, J. M., Kemper, F., \& Sloan, G. C. 2008, ApJ, 681, 1557

Lombaert, R., de Vries, B. L., de Koter, A., et al. 2012, A\&A, 544, L18

Maercker, M., Mohamed, S., Vlemmings, W. H. T., et al. 2012, Natur, 490, 232

Mainzer, A., Bauer, J., Cutri, R. M., et al. 2014, ApJ, 792, 30

Matsuura, M., Barlow, M. J., Zijlstra, A. A., et al. 2009, MNRAS, 396, 918

Matsuura, M., Bernard-Salas, J., Lloyd Evans, T., et al. 2014, MNRAS, 439, 1472

Matsuura, M., Wood, P. R., Sloan, G. C., et al. 2006, MNRAS, 371, 415

Matsuura, M., Zijlstra, A. A., van Loon, J. Th., et al. 2005, A\&A, 434, 691

McGregor, P. J., Hart, J., Hoadley, D., \& Bloxham, G. 1994, in Infrared Astronomy with Arrays, ed. I. McLean (Dordrecht: Kluwer), 299

Meixner, M., Gordon, K. D., Indebetouw, R., et al. 2006, AJ, 132, 2268

Nidever, D. L., Monachesi, A., Bell, E. F., et al. 2013, ApJ, 779, 145

Nishida, S., Tanabé, T., Nakada, Y., et al. 2000, MNRAS, 313, 136

Piatti, A. E. 2012, MNRAS, 422, 1109

Piatti, A. E., \& Giesler, D. 2013, AJ, 145, 17

Pietrzyński, G., Graczyk, D., Gieren, W., et al. 2013, Natur, 495, 76

Price, S. D., Egan, M. P., Carey, S. J., Mizuno, D. R., \& Kuchar, T. A. 2001, AJ, 121, 2819

Raimondo, G., Cioni, M.-R. L., Rejkuba, M., \& Silva, D. R. 2005, A\&A, 438, 521

Riebel, D., Boyer, M. L., Srinivasan, S., et al. 2015, ApJ, 807, 1

Riebel, D., Srinivasan, S., Sargent, B., \& Meixner, M. 2015, ApJ, 753, 71

Rubele, S., Girardi, L., Kerber, L., et al. 2015, MNRAS, 449, 639

Skrutskie, M. F., Cutri, R. M., Stiening, R., et al. 2006, AJ, 131, 1163

Sloan, G. C., \& Egan, M. P. 1995, ApJ, 444, 452

Sloan, G. C., Herter, T. L., Charmandaris, V., et al. 2015a, AJ, 149, 11

Sloan, G. C., Kraemer, K. E., Matsuura, M., et al. 2006, ApJ, 645, 1118

Sloan, G. C., Kraemer, K. E., Wood, P. R., et al. 2008, ApJ, 686, 1056
Sloan, G. C., Lagadec, E., Kraemer, K. E., et al. 2015b, in ASP Conf. Ser. 497, Why Galaxies Care about AGB Stars III, ed. F. Kerschbaum, J. Hron, \& R. Wing (San Francisco: ASP), 429

Sloan, G. C., Lagadec, E., Zijlstra, A. A., et al. 2014, ApJ, 791, 28

Sloan, G. C., Matsuura, M., Lagadec, E., et al. 2012, ApJ, 752, 140

Sloan, G. C., \& Price, S. D. 1995, ApJ, 451, 758

Soker, N., \& Livio, M. 1989, ApJ, 339, 268

Soszyński, I., Udalski, A., Szymański, M. K., et al. 2009, AcA, 59, 335

Soszyński, I., Udalski, A., Szymański, M. K., et al. 2011, AcA, 61, 217

Srinivasan, S., Boyer, M. L., Kemper, F., et al. 2016, MNRAS, 457, 2814

Srinivasan, S., Sargent, B. A., \& Meixner, M. 2011, A\&A, 532, 54

Ulaczyk, K., Szymański, M. K., Udalski, A., et al. 2013, AcA, 63, 1

van Loon, J. Th., Cohen, M., Oliveira, J. M., et al. 2008, A\&A, 487, 1055

van Loon, J. Th., Marshall, J. R., Cohen, M., et al. 2006, A\&A, 447, 971

van Loon, J. Th., Marshall, J. R., \& Zijlstra, A. A. 2005, A\&A, 442, 597

Vassiliadis, E., \& Wood, P. R. 1993, ApJ, 413, 641

Ventura, P., Karakas, A. I., Dell'Agli, F., et al. 2016, MNRAS, 457, 1456

Werner, M. W., Roellig, T. L., Low, F. J., et al. 2004, ApJS, 154, 1

Whitelock, P. A., Feast, M. W., Marang, F., \& Groenewegen, M. A. T. 2006, MNRAS, 369, 751

Whitelock, P. A., Feast, M. W., Menzies, J. W., \& Catchpole, R. M. 1989, MNRAS, 238, 769

Whitelock, P. A., Feast, M. W., van Loon, J. T., \& Zijlstra, A. A. 2003, MNRAS, 342, 86

Winters, J. M., Fleischer, A. J., Gauger, A., \& Sedlmayr, E. 1994, A\&A, 290, 623

Woitke, P. 2006, A\&A, 452, 537

Wright, E. L., Eisenhardt, P. R. M., Mainzer, A. K., et al. 2010, AJ, 140, 1868

Zaritsky, D., Harris, J., Thompson, I. B., \& Grebel, E. K. 2004, AJ, 128, 1606

Zaritsky, D., Harris, J., Thompson, I. B., Grebel, E. K., \& Massey, P. 2002, AJ, 123,855

Zhang, K., Jiang, B. W., \& Li, A. 2009, ApJ, 702, 680

Zijlstra, A. A., Matsuura, M., Wood, P. R., et al. 2006, MNRAS, 370, 1961 